\title{
Transdermal Administration of Volatile Oil from Citrus aurantium-Rhizoma Atractylodis Macrocephalae Alleviates Constipation in Rats by Altering Host Metabolome and Intestinal Microbiota Composition
}

\author{
Liangfeng Wang, ${ }^{1,2}$ Fang Wang $\mathbb{D}^{1},{ }^{1}$ Xiaofei Zhang, ${ }^{1,3}$ Qingyao Chen, ${ }^{1}$ Jie Xu, ${ }^{1}$ Huiting Li, \\ FengQin Li, ${ }^{1}$ and Ming Yang ${ }^{1}$ \\ ${ }^{1}$ Key Laboratory of Modern Preparation of Traditional Chinese Medicine, Ministry of Education, Jiangxi University of \\ Chinese Medicine, Nanchang 30004, China \\ ${ }^{2}$ College of Chinese Materia Medica, Shanghai University of Traditional Chinese Medicine, Shanghai 201203, China \\ ${ }^{3}$ College of Pharmacy, Shanxi University of Chinese Medicine, Shanxi 712046, China
}

Correspondence should be addressed to Fang Wang; wangfang09421@126.com and Ming Yang; yangming16@126.com

Received 13 August 2021; Revised 9 October 2021; Accepted 18 October 2021; Published 18 January 2022

Academic Editor: Nattaya Konsue

Copyright (c) 2022 Liangfeng Wang et al. This is an open access article distributed under the Creative Commons Attribution License, which permits unrestricted use, distribution, and reproduction in any medium, provided the original work is properly cited.

Background. The Citrus aurantium- (ZhiShi, ZS-) Rhizoma Atractylodis Macrocephalae (BaiZhu, BZ) pairs are often found in herbal formulas for constipation. The volatile oils of ZS and BZ (ZBVO) have good pharmacological activity against constipation, but the mechanism for treatment of slow transit constipation (STC) remains unclear. Method. A rat model using diphenoxylate tablets was constructed to investigate if transdermal administration of ZBVO would mediate intestinal microorganisms and fecal metabolites and improve STC symptoms. The regulatory effects of ZBVO at $0.15,0.30$, and $0.60 \mathrm{~mL} \mathrm{~kg}^{-1} \mathrm{~d}^{-1}$ on STC rats were assessed by measuring fecal water content, intestinal propulsion rate, histopathology, expression of gastrointestinal hormones, brain and intestinal peptides, and inflammatory factors. The changes in intestinal flora of STC rats were analyzed by $16 \mathrm{~S}$ rRNA gene sequencing. Moreover, the untargeted fecal metabolomics analysis was performed by ultraperformance liquid chromatography quadrupole time-of-flight mass spectrometer (UPLC-Q-TOF-MS) technology. Results. The results showed that ZBVO had a modulating effect on STC by increasing the fecal water content and intestinal propulsion rate. Transdermal administration of ZBVO decreased serum levels of interleukin 6 (IL-6) and tumor necrosis factor- $\alpha$ (TNF- $\alpha$ ) and increased the levels of gastrin (GAS) and substance P (SP). In addition, ZBVO increased 5hydroxytryptamine (5-HT) levels and decreased vasoactive intestinal peptide (VIP) levels in colon and hippocampus tissues. The results of intestinal microbiota showed that ZBVO improved the diversity and abundance of intestinal microbiota and changed the community composition by decreasing Romboutsia and increasing Proteobacteria, Allobaculum, and Ruminococcaceae. And the feces metabolomics found that nicotinate and nicotinamide metabolism, purine metabolism, citrate cycle (TCA cycle), pyruvate metabolism, arachidonic acid metabolism, pyrimidine metabolism, and primary bile acid biosynthesis were modulated. Conclusion. These findings suggest that ZBVO can alleviate STC symptoms by promoting intestinal peristalsis, increasing fecal water content, regulating gastrointestinal hormone level, reducing the inflammatory response, and regulating brain and intestinal peptides after transdermal administration. And structural changes in the intestinal microbiota are closely related to host metabolism and intestinal microbiota destroyed in STC modeling could be significantly improved by the ZBVO, which provides a reference for the development of aromatic drug macrohealth products. 


\section{Introduction}

Slow transit constipation (STC) is a common form of functional constipation characterized by reduced stool frequency, loss of bowel movement, dry and hard stools, and abdominal distension [1,2]. Epidemiological studies indicate the global prevalence of STC is $14 \%$ [3], which seriously affects the physical and mental health of patients [4]. In addition, STC is closely associated with abdominal distension, cardiovascular and anal diseases, colorectal cancer, and Alzheimer's disease [5]. The etiology of STC is complex, and its mechanisms are not fully understood. The etiology of STC is mainly related to the enteric nervous system, gastrointestinal hormones, neurotransmitters, and intestinal microbiota $[6,7]$. Currently, the main treatment methods for STC include medication and biofeedback therapy that often cause adverse reactions and drug dependence and highly invasive surgery that can have serious postoperative complications, such as abdominal pain and diarrhea $[8,9]$. Therefore, natural medicines could offer better efficacy and applicability.

In most cases, a single drug does not completely cure STC; consequently, drug combinations are often used to exert synergistic effects and improve efficacy or reduce adverse side effects. STC has multiple causative factors. According to traditional Chinese medicine (TCM), the main cause is abnormal conduction of the large intestine, poor Qi flow, and internal stagnation of dregs and is closely related to the dysfunction of the spleen and stomach. In clinical practice, herbs that strengthen the spleen and regulate Qi are commonly used to treat STC. Citrus aurantium- (ZS-) Rhizoma Atractylodis Macrocephalae (BZ) is a classical drug pair that is recorded in many traditional formulas, such as Zhizhu decoction, and is commonly used to treat STC Jinkui Yaolue. ZS is a classical Qi-regulating herb that can treat stool obstruction and has the ability to break up Qi and eliminate stagnation and dissolve and disperse phlegm. ZS and its volatile oil have a long history of clinical application and are often used in TCM to treat stool obstruction, likely by enhancing the colon propulsion function and correcting the abnormal colonic slow waves of STC [10]. D-Limonene, the main component of ZS volatile oil, also has antibacterial activity [11]. BZ has the effect of replenishing Qi and tonifying the spleen, removing water from the intestinal track, and promoting water circulation. BZ volatile oil has a regulatory effect on gastrointestinal motility and body immunity, in addition to antibacterial, anti-inflammatory, and antiaging effects [12]. Its main active ingredient, atractylon, even has a laxative effect [13].

A transdermal drug delivery system not only avoids the first-pass effect but also is painless, aids patients with swallowing difficulties, increases patient compliance, and can be self-administered. The Shenque point means "Spirit Gate," which is in the center of the abdomen and is a point that represents the final closure of the abdominal wall at the end of embryonic development. The Shenque point has a thin cuticle making it conducive to rapid drug absorption, and its location close to the intestinal tract strengthens the spleen and stomach by promoting the flow of Qi and relaxing the bowels [14]. This study takes the advantages of strong fast penetration and easy dispersal of Chinese medicine volatile oil, which can be absorbed through transdermal administration at the umbilicus, to adjust gastrointestinal dynamics, promote recovery of spleen and stomach functions, and achieve the therapeutic effect of moving Qi and laxative. However, the mechanism by transdermal administration of ZBVO improving STC remains unclear, therefore making it crucial to elucidate the basic mechanism of action in order to provide a new effective drug and treatment method for STC.

Intestinal microbiota has been implicated in the pathogenesis of various human diseases, such as irritable bowel syndrome (IBS), cardiovascular disease, and inflammatory bowel disease $[15,16]$. Moreover, changes in intestinal microbiota composition are related to the pathogenesis of STC [17]. Dysbiosis of the intestinal microbiota may affect the brain-gut axis, thus promoting brain-gut peptide disorders. 16S rRNA gene sequencing studies enable accurate identification of the structure of intestinal microorganisms [18]. Metabolomics can comprehensively reflect the changes of metabolites in the process of disease occurrence and development [19]. Together, 16S rRNA gene sequencing and metabolomics have been widely used in the study of disease and drug treatment mechanisms, to reveal the pathogenic mechanism of diseases [20] and screening for biomarkers of diseases. However, the specific metabolites by which ZBVO affects host fecal metabolism via intestinal microbes are still unclear.

In the current study, we investigated the effects of $\mathrm{ZBVO}$ on the intestinal microbiota and fecal metabolite composition of STC rats induced by compound diphenoxylate tablets. To this end, we used 16S rRNA gene sequencing and ultraperformance liquid chromatography quadrupole timeof-flight mass spectrometer- (UPLC-Q-TOF-MS-) based metabolomics to determine the effects of $\mathrm{ZBVO}$ on the gut microbial community structure and fecal metabolomics of STC rats after transdermal administration via the umbilicus.

\section{Materials and Methods}

2.1. Materials and Chemicals. Compound diphenoxylate tablets (batch number: 1712029) were purchased from Changzhou Kangpu Pharmaceutical Co., Ltd. (Jiangsu, China). Mosapride (batch number: 190201) was purchased from Jiangsu Haosen Pharmaceutical Co., Ltd. (Jiangsu, China). The commercial enzyme-linked immunosorbent assay (ELISA) kits for tumor necrosis factor- $\alpha$ (TNF- $\alpha$ ), interleukin 6 (IL-6), substance P (SP), gastrin (GAS), 5hydroxytryptamine (5-HT), and vasoactive intestinal peptide (VIP) were purchased from Jiangsu Enzymatic Immunity Industry Co., Ltd. (Yancheng, China). Neutral gum and phosphate-buffered saline (PBS) solution were obtained from Beijing Solarbio Science \& Technology Co., Ltd. (Beijing, China). HPLC-grade methanol, acetonitrile, isopropanol, ammonium hydroxide, and pure distilled water were obtained from Fisher Scientific (Fair Lawn, NJ, USA).

2.2. Plant Materials and Volatile Oil Extraction. Citrus aurantium (also known as ZhiShi in China) and Rhizoma 
Atractylodis Macrocephalae (also known as BaiZhu in China) were purchased from Chengdu Huichu Technology Co., Ltd. (Chengdu, China), which were authenticated by Professor Fei Ge (Jiangxi University of Traditional Chinese Medicine, Nanchang, China). According to the ratio of ZS $: \mathrm{BZ}=2: 1$, the dried Chinese herbs were crushed into coarse powder. The herbs were distilled in seven volumes of water for 8 hours using an oil-water separator to produce ZBVO. The volatile oil samples were precisely absorbed from $100 \mu \mathrm{L}$ to $10 \mathrm{~mL}$ capacity flasks after anhydrous $\mathrm{Na}_{2} \mathrm{SO}_{4}$ dehydration, volume fixation with $\mathrm{n}$-hexane, and passed through $0.22 \mu \mathrm{m}$ microporous filter membrane. Gas chromatography-mass spectrometry (GC-MS) was used to control the quality of ZBVO. The gas chromatographic conditions were as follows: Agilent DB-624 (30 $\mathrm{m} \times 250 \mu \mathrm{m}$, $0.25 \mu \mathrm{m})$ capillary column, high-purity He (99.999\%) carrier gas, and $1 \mu \mathrm{L}$ sample volume was used. The split ratio was nonshunt, and the flow rate was $1 \mathrm{~mL} / \mathrm{min}$. The temperature program was as follows: initial temperature of $40^{\circ} \mathrm{C}$ (held for $1 \mathrm{~min}$ ), ramp to $10^{\circ} \mathrm{C} / \mathrm{min}$ to $220^{\circ} \mathrm{C}$, and then increased to $25^{\circ} \mathrm{C} / \mathrm{min}$ to $280^{\circ} \mathrm{C}$ (held for $9 \mathrm{~min}$ ). Mass spectrometry conditions were as follows: EI ion source, electron energy $70 \mathrm{eV}$, ion source temperature $230^{\circ} \mathrm{C}$, MS quadrupole temperature $150^{\circ} \mathrm{C}$, interface temperature $250^{\circ} \mathrm{C}$, solvent delay $3.0 \mathrm{~min}$, quality scan pattern full scan, and scan range of 30 650 amu. Standard spectral library NIST11 retrieval, peak area normalization method was used to calculate the relative percentage content of each component.

2.3. Animal Experimental Design. Sprague Dawley (SD) rats with a bodyweight of $200 \pm 20 \mathrm{~g}$ were obtained from the Hunan Shrek Jingda Experimental Animal Co., Ltd. (Hunan, China; certificate number SCXK 2019-0004). The rats were housed at a standard laboratory environment (ambient temperature: $23 \pm 1^{\circ} \mathrm{C} ; 12 \mathrm{~h}$ light/dark cycle; relative humidity: $40 \%-70 \%)$ for one week with free access to food and water. All experimental procedures were approved by the Animal Ethics Committee of Jiangxi University of TCM and conformed to internationally accepted guidelines for the use of experimental animals.

After a week of adaptive feeding, the rats (24 male and 24 female) were randomly divided into six groups (four male and four female rats in each group): blank control (BC) group, model control (MC) group, low-dose (LD) treatment group, middle-dose (MD) treatment group, high-dose (HD) treatment group, and positive control (PC) group. The diphenoxylate-induced mouse STC model was established as previously described, with minor modifications [21]. After acclimation to the conditions for 1 week, except for the $\mathrm{BC}$ group, rats were treated with diphenoxylate $(10 \mathrm{~mL} / \mathrm{kg})$ solution soluble in $0.5 \%$ sodium carboxymethyl cellulose (CMC-Na) by oral gavage once a day for 28 days. Starting on the seventh day, the $\mathrm{LD}, \mathrm{MD}$, and $\mathrm{HD}$ groups had the appropriate dosage $\left(0.15,0.3\right.$, and $0.6 \mathrm{~mL} \mathrm{~kg}^{-1} \mathrm{~d}^{-1}$, respectively) diluted with jojoba oil at 10 times the dosage, smeared on the Shenque point (the Shenque point is located one-third of the way down from the line connecting the symphysis of the rat's saber and pubic bone) for 21 days. Rats in the PC group were given mosapride $\left(1.5 \mathrm{mg} \mathrm{kg}^{-1} \mathrm{~d}^{-}\right.$ $\left.{ }^{1}\right)$ solution soluble in $0.5 \% \mathrm{CMC}-\mathrm{Na}$ by intragastric administration once a day for 21 days. The rats in the $\mathrm{BC}$ group were treated with the same volume of CMC-Na solution by oral gavage once a day for 28 days (see Figure 1(a)).

2.4. Sample Collection. At the end of the intervention, on the $28^{\text {th }}$ day, 2 hours after the final administration, fresh fecal samples were collected and stored at $-80^{\circ} \mathrm{C}$. DNA was immediately extracted from fecal samples for microbiome analysis. All rats were fasted overnight $(12 \mathrm{~h})$ and then given $2 \mathrm{~mL}$ activated carbon suspension by intragastric administration. Forty minutes later, they were lightly anesthetized by intravenous injection of $1 \%$ pentobarbital ( $40 \mathrm{mg}$ pentobarbital/kg rat bodyweight) and blood was collected from the abdominal aorta. The blood was centrifuged at $3000 \mathrm{rpm}$ for $10 \mathrm{~min}$ at $4^{\circ} \mathrm{C}$ to obtain serum. The sera were stored at $-80^{\circ} \mathrm{C}$ until analyzed. The small intestines, from pylorus to lower caecum, were extracted from every rat. At the same time, the colon tissue was cut open quickly; part of the colon was frozen in liquid nitrogen and then preserved at $-80^{\circ} \mathrm{C}$, while the remaining portion was fixed in $4 \%$ paraformaldehyde for further study.

2.5. Fecal Water Content and Intestinal Propulsion Rate. In this study, the water content in rat feces was calculated as described earlier [22]. In short, the feces of rats in each group were collected and dried at $50^{\circ} \mathrm{C}$ oven for 4 hours, and the fecal water content was calculated as follows:

$$
\text { fecal water content }=\left(\frac{\text { wet weight }- \text { dry weight }}{\text { wet weight }}\right) \times 100 \% \text {. }
$$

A scrape from the small intestine (the front end of the cecum) was placed on a clean glass plate without traction. We measured the total length and the distance from the front end of the carbon powder to the pylorus to calculate the ratio to the total length:

$$
\begin{aligned}
& \text { intestinal propulstion rate } \\
& \qquad=\left(\frac{\text { charcoal powder propulsion distance }(\mathrm{cm})}{\text { intestinal full length }(\mathrm{cm})}\right) \times 100 \% \text {. }
\end{aligned}
$$

2.6. Determination of Gastrointestinal Hormones (GAS and $S P$ ) and Inflammatory Factors (TNF- $\alpha$ and IL-6). The serum was taken and the contents of GAS, SP, IL-6, and TNF- $\alpha$ were determined by using ELISA kits accordingly, and the absorbance was set at $450 \mathrm{~nm}$ and measured on a microplate reader.

2.7. Determination of Brain-Gut Peptide (5-HT and VIP). Colon and hippocampal tissues were homogenized with PBS at a ratio of $1: 9$ (weight: volume) on ice using a glass homogenizer, respectively. The supernatant was obtained by centrifugation at $3500 \mathrm{rpm} / \mathrm{min}$ at $4^{\circ} \mathrm{C}$ for $10 \mathrm{~min}$. The total protein in the sample was detected with a BCA kit, and the content of 5-HT and VIP in the colon and 


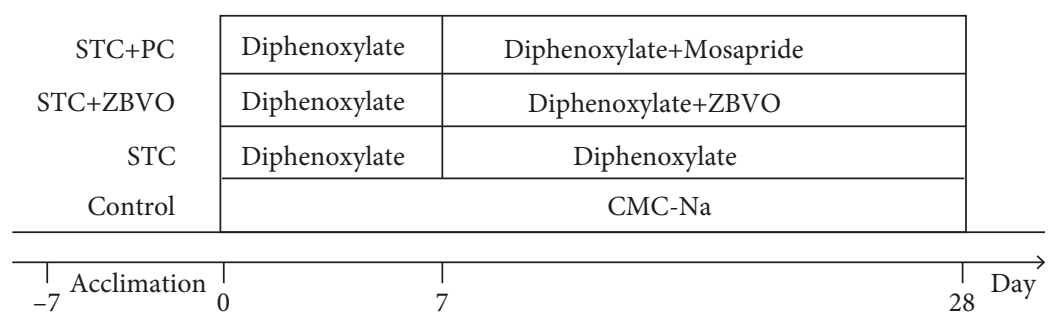

(a)

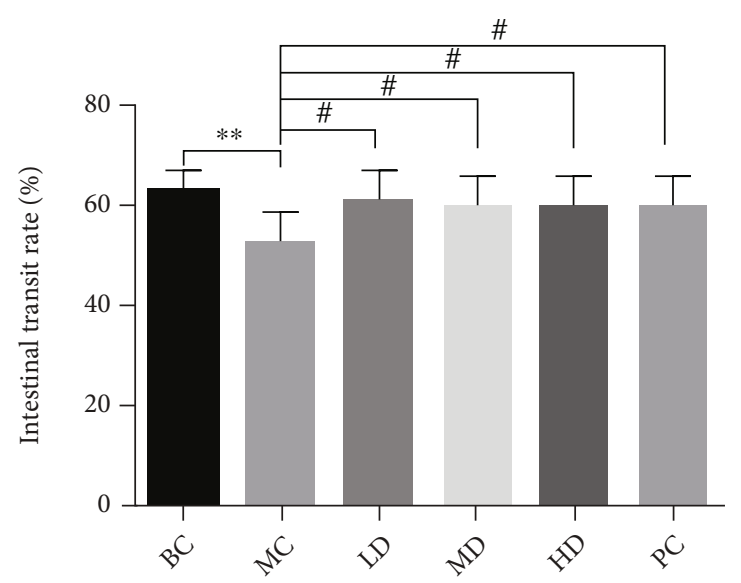

(b)

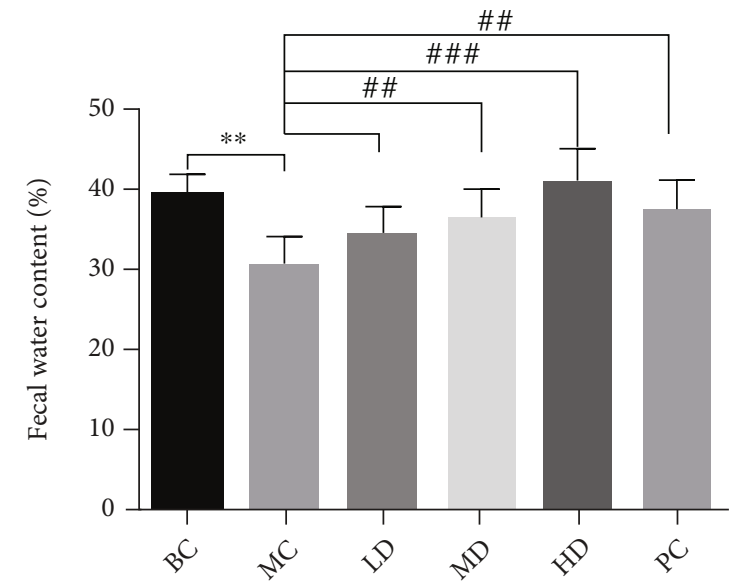

(c)

FIGURE 1: Effect of ZBVO on improving the fecal water content and intestinal transit rate of STC rats induced by compound diphenoxylate tablets $(n=8)$. (a) Schematic diagram of STC model establishment and transdermal drug administration treatment. (b) ZBVO improved intestinal propulsion rate and intestinal transit in diphenoxylate-induced STC rats $\left(150,300\right.$, and $600 \mu \mathrm{Lkg}^{-1} \mathrm{~d}^{-1}$ ) and mosapride. (c) Compared with the MC group, ZBVO dosing $\left(150,300\right.$, and $\left.600 \mu \mathrm{L} \mathrm{kg}^{-1} \mathrm{~d}^{-1}\right)$ increased fecal water content in rats. Values are expressed as mean \pm SEM. ${ }^{*} p<0.05,{ }^{* *} p<0.01$, and ${ }^{* * *} p<0.001$ vs. the BC group. ${ }^{\#} p<0.05,{ }^{\# \#} p<0.01$, and ${ }^{\# \# \#} p<0.001$ vs. the MC group.

hippocampal tissues was determined by using ELISA kits accordingly. The 5-HT and VIP contents were measured at $450 \mathrm{~nm}$ on a microplate reader. Finally, the fruit value was calculated as

$$
\text { fruit value }=\frac{\text { ELISA concentration value }}{\text { BCA concentration value }} \text {. }
$$

2.8. Pathological Evaluation. The rat colon was fixed in $4 \%$ paraformaldehyde for 24 hours, routinely dehydrated, embedded, sectioned, and stained with hematoxylin-eosin stain according to the standard experimental procedure. The pathological histological changes were observed under a light microscope with a $\times 100$ objective.

2.9. 16S rRNA Gene-Based Bacterial Community Analysis. Community structure of rat intestinal microbiota was analyzed by $16 \mathrm{~S}$ rRNA gene sequencing technique. A genomic DNA isolation kit (MoBio Laboratories, Carlsbad, CA) was used to extract total DNA samples from the rat feces samples. The extracted DNA was detected by $1 \%$ agarose gel electrophoresis, and quality and concentration were determined using spectrophotometry. Paired-end sequencing was performed using the Illumina MiSeq PE300 highthroughput sequencing platform at Beijing Aoweisen Gene Technology Co., Ltd. The primers 515F ( $5^{\prime}$-GTGCCAGC
MGCCGCGGTAA-3') and 806R ( $5^{\prime}$-GGACTACHVGGG TWTCTAAT- $3^{\prime}$ ) were used to amplify the bacterial $16 \mathrm{~S}$ rRNA gene V4 region. The PCR products were detected by $1 \%$ agarose gel electrophoresis for amplification target band size and purified with an Agencourt AMPure XP Nucleic Acid Purification Kit. The raw sequences were uploaded to NCBI's SRA database. Pairs of reads were spliced into one sequence, and the data were optimized for OTU (97\% similarity level) clustering analysis using the RDP Classifier algorithm. Diversity analysis and multilevel discriminate analysis size effect (LEfSe) were performed on all samples; environmental factor correlation analysis was performed using the Spearman correlation analysis.

2.10. UPLC-Q-TOF-MS Analysis. A $30 \mathrm{mg}$ rat fecal sample was placed into a $2 \mathrm{~mL}$ EP tube, and then, $500 \mu \mathrm{L}$ of extraction solution was added (methanol : acetonitrile : water $=2$ $: 2: 1$ ). Samples were processed using a $60 \mathrm{~Hz}$ grinder for $2 \mathrm{~min}$, sonicated in an ice water bath for $10 \mathrm{~min}$, and kept at $-20^{\circ} \mathrm{C}$ for $1 \mathrm{~h}$. Samples were centrifuged at $13,000 \mathrm{r} / \mathrm{min}$ for $15 \mathrm{~min}$ at $4^{\circ} \mathrm{C}$, and then, $350 \mu \mathrm{L}$ of supernatant was aspirated and placed in a vacuum freeze dryer until evaporated and dry. Then, $100 \mu \mathrm{L}$ of extraction solution (acetonitrile : water $=1: 1$ ) was added to the dried metabolites for resolubilization and vortexed for $30 \mathrm{~s}$, followed by 
TABLE 1: Main chemical composition and percentage of ZBVO.

\begin{tabular}{|c|c|c|c|c|c|}
\hline No. & $\begin{array}{l}\text { Retention time } \\
\quad(\min )\end{array}$ & Compound & $\begin{array}{l}\text { Chemical } \\
\text { formula }\end{array}$ & $\begin{array}{c}\text { Relative } \\
\text { amount (\%) }\end{array}$ & CAS \\
\hline 1 & 7.337 & $\beta$-Pinene & $\mathrm{C}_{10} \mathrm{H}_{16}$ & 1.18 & $000127-91-3$ \\
\hline 2 & 7.520 & $\beta$-Myrcene & $\mathrm{C}_{10} \mathrm{H}_{16}$ & 0.32 & 000123-35-3 \\
\hline 3 & 8.280 & D-Limonene & $\mathrm{C}_{10} \mathrm{H}_{16}$ & 42.72 & 005989-27-5 \\
\hline 4 & 8.470 & $\beta$-Ocimene & $\mathrm{C}_{10} \mathrm{H}_{16}$ & 0.55 & 013877-91-3 \\
\hline 5 & 8.912 & Linalyl oxide & $\mathrm{C}_{10} \mathrm{H}_{18} \mathrm{O}_{2}$ & 0.19 & 005989-33-3 \\
\hline 6 & 9.170 & Terpinolene & $\mathrm{C}_{10} \mathrm{H}_{16}$ & 0.17 & $000586-62-9$ \\
\hline 7 & 9.333 & Linalool & $\mathrm{C}_{10} \mathrm{H}_{18} \mathrm{O}$ & 1.34 & 000078-70-6 \\
\hline 8 & 10.616 & Terpinen-4-ol & $\mathrm{C}_{10} \mathrm{H}_{18} \mathrm{O}$ & 0.39 & 000562-74-3 \\
\hline 9 & 10.819 & $(-)-\beta$-Fenchyl alcohol & $\mathrm{C}_{10} \mathrm{H}_{18} \mathrm{O}$ & 0.45 & 000470-08-6 \\
\hline 10 & 11.240 & (-)-trans-Carveol & $\mathrm{C}_{10} \mathrm{H}_{16} \mathrm{O}$ & 0.35 & 001197-07-5 \\
\hline 11 & 11.417 & Carveol & $\mathrm{C}_{10} \mathrm{H}_{16} \mathrm{O}$ & 0.17 & $000099-48-9$ \\
\hline 12 & 11.620 & d-Carvone & $\mathrm{C}_{10} \mathrm{H}_{14} \mathrm{O}$ & 0.15 & 002244-16-8 \\
\hline 13 & 12.645 & Dodecamethylcyclohexasiloxane & $\mathrm{C}_{12} \mathrm{H}_{36} \mathrm{O}_{6} \mathrm{Si}_{6}$ & 0.14 & 000540-97-6 \\
\hline 14 & 12.978 & $\alpha$-Terpinene & $\mathrm{C}_{15} \mathrm{H}_{24}$ & 0.38 & 000099-86-5 \\
\hline 15 & 13.752 & $\beta$-Elemene & $\mathrm{C}_{15} \mathrm{H}_{24}$ & 0.52 & $000515-13-9$ \\
\hline 16 & 13.942 & (-)-Cyperene & $\mathrm{C}_{15} \mathrm{H}_{24}$ & 0.17 & $002387-78-2$ \\
\hline 17 & 14.193 & $\beta$-Caryophyllene & $\mathrm{C}_{15} \mathrm{H}_{24}$ & 1.08 & $000087-44-5$ \\
\hline 18 & 14.336 & $\gamma$-Elemene & $\mathrm{C}_{15} \mathrm{H}_{24}$ & 7.83 & 029873-99-2 \\
\hline 19 & 14.655 & Humulene & $\mathrm{C}_{15} \mathrm{H}_{24}$ & 0.47 & 006753-98-6 \\
\hline 20 & 14.899 & $\gamma$-Cadinene & $\mathrm{C}_{15} \mathrm{H}_{24}$ & 0.32 & 030021-74-0 \\
\hline 21 & 15.008 & Germacrene D & $\mathrm{C}_{15} \mathrm{H}_{24}$ & 2.05 & 023986-74-5 \\
\hline 22 & 15.089 & $\beta$-Selinene & $\mathrm{C}_{15} \mathrm{H}_{24}$ & 1.34 & $017066-67-0$ \\
\hline 23 & 15.184 & Valencene & $\mathrm{C}_{15} \mathrm{H}_{24}$ & 0.32 & 004630-07-3 \\
\hline 24 & 15.483 & (+)-delta-Cadinene & $\mathrm{C}_{15} \mathrm{H}_{24}$ & 0.62 & 000483-76-1 \\
\hline 25 & 15.714 & Isoledene & $\mathrm{C}_{15} \mathrm{H}_{24}$ & 3.71 & 095910-36-4 \\
\hline 26 & 15.802 & $\begin{array}{c}\left.\text { 3-(2', } 4^{\prime} \text {-Dimethylpenta-1' }{ }^{\prime} 4^{\prime} \text {-dien- } 3^{\prime} \text {-ylidene }\right)-6,6 \text {-dimethylcyclohex- } \\
\text { 1-ene }\end{array}$ & - & 0.76 & 997214-07-9 \\
\hline 27 & 15.890 & Nerolidol & $\mathrm{C}_{15} \mathrm{H}_{26} \mathrm{O}$ & 0.20 & $007212-44-4$ \\
\hline 28 & 16.067 & Isolongifolene, 9,10-dehydro- & $\mathrm{C}_{15} \mathrm{H}_{22}$ & 0.85 & $997214-17-0$ \\
\hline 29 & 16.630 & 4-Bromo-1-naphthylamine & $\mathrm{C}_{10} \mathrm{H}_{8} \mathrm{BrN}$ & 0.23 & 002298-07-9 \\
\hline 30 & 16.841 & Spathulenol & $\mathrm{C}_{15} \mathrm{H}_{24} \mathrm{O}$ & 1.01 & $006750-60-3$ \\
\hline 31 & 17.296 & Atractylon & $\mathrm{C}_{15} \mathrm{H}_{20} \mathrm{O}$ & 27.51 & $006989-21-5$ \\
\hline 32 & 17.526 & Vulgarol B & $\mathrm{C}_{20} \mathrm{H}_{36} \mathrm{O}_{2}$ & 0.22 & 011056-03-4 \\
\hline 33 & 17.662 & $\begin{array}{l}\text { (1R,4Ar,8aR)-1,4a-Dimethyl-7-propan-2-ylidene-3,4,5,6,8,8a- } \\
\text { hexahydro-2H-naphthalen-1-ol }\end{array}$ & $\mathrm{C}_{15} \mathrm{H}_{26} \mathrm{O}$ & 0.15 & 000473-04-1 \\
\hline 34 & 17.839 & $\begin{array}{l}\text { 1-Hydroxy-1-methyl-7(methylethenyl) }[1,2,3,3 a, 4,5,6,7] \text { octahydro } \\
\text { azulene }\end{array}$ & - & 0.17 & $997228-20-5$ \\
\hline 35 & 18.083 & 4-Methoxy-5-oxidanyl-naphthalene-2-carboxylic acid & - & 0.72 & $997266-09-1$ \\
\hline 36 & 19.889 & 1,5,9-Trimethyl-2-oxatricyclo[7.3.0.0(3,8)]dodec-3(8),4,6-triene & - & 0.82 & $997213-83-9$ \\
\hline 37 & 20.106 & Palmitic acid & $\mathrm{C}_{16} \mathrm{H}_{32} \mathrm{O}_{2}$ & 0.30 & 000057-10-3 \\
\hline 38 & 20.737 & 3-Phenyl-1,2-dihydrocyclopenta[a]indene & $\mathrm{C}_{18} \mathrm{H}_{14}$ & 0.16 & $997312-45-2$ \\
\hline
\end{tabular}

10 min of sonication in an ice-water bath. We then centrifuged at $13,000 \mathrm{r} / \mathrm{min}$ for another $15 \mathrm{~min}$ at $4^{\circ} \mathrm{C}$. Next, we placed $50 \mu \mathrm{L}$ of the resulting supernatant into the injection bottle and waited for UPLC-Q-TOF-MS detection. The samples were separated in Waters Acquity UPLC BEH Amide $(2.1 \times 100 \mathrm{~mm}, 1.7 \mu \mathrm{m})$. The mobile phase consisted of $(\mathrm{A})$ ultrapure water (containing $25 \mathrm{mM}$ ammonium acetate and $25 \mathrm{mM}$ ammonium hydroxide) and (B) acetonitrile. The elution gradient is shown in Supplemental Table 1. The flow rate was $0.5 \mathrm{~mL} / \mathrm{min}$, column temperature was $40^{\circ} \mathrm{C}$, and the injection volume was $2 \mathrm{~mL}$. Briefly, the mass spectrometry conditions were set as follows: electrospray ion source temperature (TEM) of $650^{\circ} \mathrm{C}$; mass spectrometry voltage (ISVF) $5500 \mathrm{~V}$ (positive ions), $-4500 \mathrm{~V}$ (negative ions); declustering voltage (DP) $60 \mathrm{~V}$; ion source gas $60 \mathrm{psi}$ for both gas 1 and gas 2, gas curtain (CUR) 30psi; 


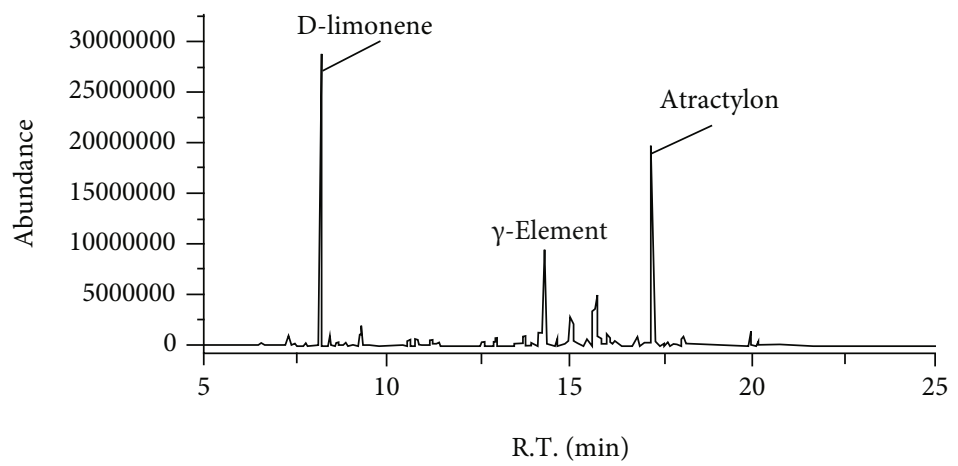

(a)

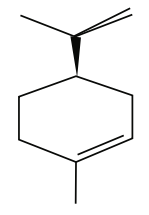

D-limonene

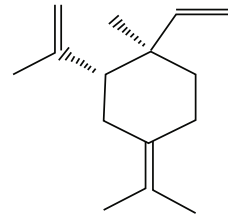

$\gamma$-Elemene<smiles>C=C1CCC[C@]2(C)CC3=C(C[C@H]12)C(C)CO3</smiles>

Atractylon

(b)

FIGURE 2: GC-MS analysis of ZBVO. (a) GC-MS analysis of the TIC component of ZBVO. (b) Structures of three main chemical compounds isolated from ZBVO.

atomization pressure (GS1) $60 \mathrm{~Pa}$, auxiliary pressure $60 \mathrm{~Pa}$, spray voltage $5500 \mathrm{~V}$ (positive ion mode) or $-4500 \mathrm{~V}$ (negative ion mode), mass spectrometry scan range MS1 60$1200 \mathrm{Da}, \mathrm{MS} 2$ 25-1200 Da.

2.11. Statistical Analysis. Statistical analyses were performed using IBM SPSS Statistics 21.0 (Chicago, USA). The experimental data in this study are presented as mean \pm standard deviation (SD). Single-factor ANOVA was used for analysis, LSD method was used for homogeneity of variance, GamesHowell method was used for heterogeneity of variance, and $p<0.05$ was considered a significant difference and $p<$ 0.01 was considered a very significant difference.

MarkerView 1.3.1 software was used for peak identification, peak extraction, and peak alignment, while minfrac and cutoff were set to 0.5 and 0.6 , respectively. Substance identification of peaks was performed by secondary mass spectrometry database, $\mathrm{R}$ program package. The missing values that appear in the original data are simulated, and the simulation method is the minimum value 1:2 method for filling. The low-quality ions were removed according to the " $80 \%$ principle," and finally, the peak area was normalized using the total ion flow of each sample. Univariate Student's $t$ -test between the blank group and the STC model group was performed, and statistical significance was considered as $p<0.05$. Multivariate analyses, including unsupervised PCA and supervised OPLS-DA analysis, were performed using SIMCA 14.1 software, and the model was validated. Statistical significance was considered as $p<0.05$ in the $t$-test, and VIP $\geq 1$ was used to screen for difference variables. Human metabolome database (HMDB, http://www .hmdb.ca) and the Kyoto Encyclopedia of genes and genomes (KEGG, http://www.kegg.jp) database were selected for metabolite identification, and the identified differential metabolites were analyzed by the Pathway Analysis module in the MetaboAnalyst 3.0 platform for metabolic pathway analysis with the pathway impact $>0$ and $-\log (p)>0.5$ set as screening conditions.

\section{Results}

3.1. Composition Analysis of $Z B V O$. The main chemical composition and relative percentage of ZBVO analyzed by GC-MS are shown in Table 1 . A total of 38 chemical components were identified, with the majority being D-limonene $(42.72 \%)$, followed by atractylon $(27.51 \%)$, and then $\gamma$-elemene $(7.83 \%)$. GC-MS analysis of the Total Ion Chromatography (TIC) component of ZBVO is shown in Figure 2(a). Structures of D-limonene, atractylon, and $\gamma$-elemene are presented in Figure 2(b).

3.2. Effects of $Z B V O$ on Intestinal Motility and Fecal Water Content. Figure 1 shows the effects of ZBVO on intestinal motility and fecal water content in STC rats. In Figure 1(b), the MC group had significantly lower intestinal motility velocity than the $\mathrm{BC}$ group $(p<0.01)$, indicating that the STC model was successfully induced. All three doses of ZBVO and mosapride were effective in promoting intestinal motility in STC rats $(p<0.05)$. In Figure $1(c)$, the MC group showed a significant decrease in fecal water content compared to the BC group $(p<0.001)$, while ZBVO transdermal administration showed a dose-dependent increase in fecal water content $(p<0.01)$. These results suggest that ZBVO treatment effectively promoted defecation and enhanced colonic dynamics in rats. 


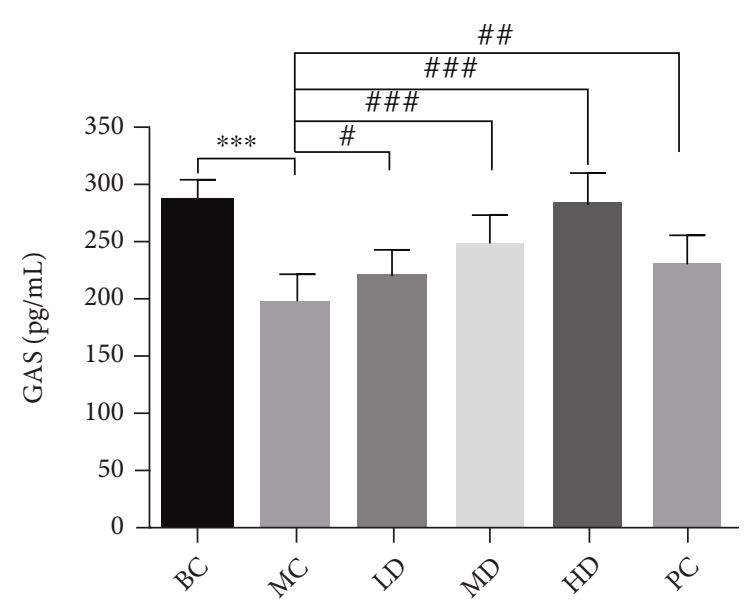

(a)

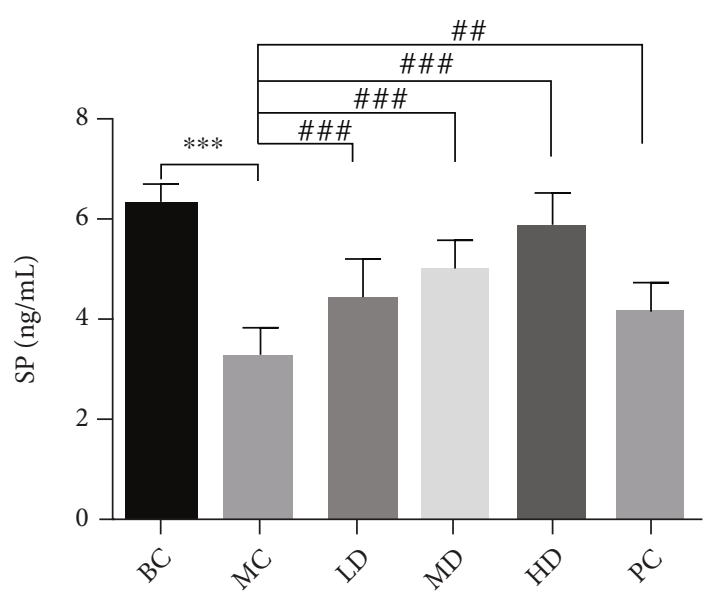

(b)

Figure 3: Effects of ZBVO treatment on (a) GAS and (b) SP levels in the serum $(n=8)$. Compared with the normal group: ${ }^{* *} p<0.01$ and ${ }^{*} p<0.05$; compared with the model group: ${ }^{\# \#} p<0.01$ and ${ }^{\#} p<0.05$.
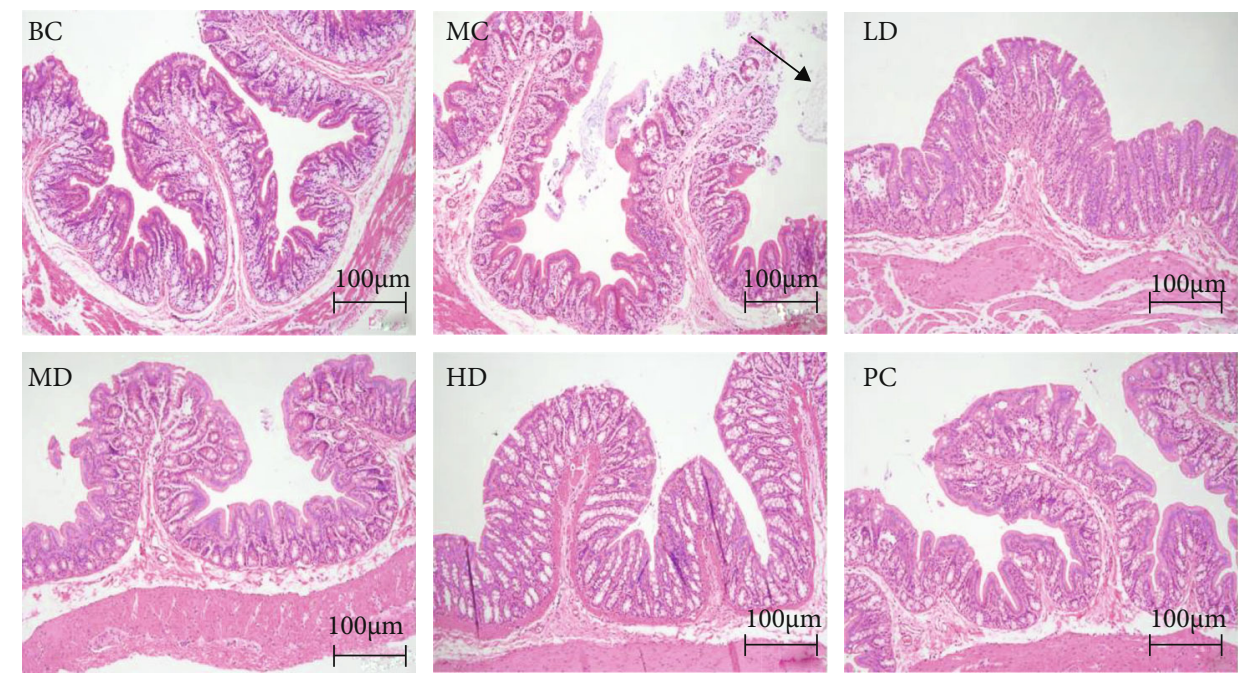

FIGURE 4: Histopathology examination of the colon in STC rats. H\&E staining of the colon $(n=3)$.

3.3. Effects of ZBVO on Gastrointestinal Hormones (GAS and SP) in the Serum, Pathological Histology of Colonic Tissue, and Inflammatory Factors (TNF- $\alpha$ and IL-6) in the Serum. Assessment of serum levels of gastrointestinal hormones and inflammatory factors can be used to reveal the role of ZBVO in STC regulation. GAS and SP levels in the MC group were significantly lower than those in the $\mathrm{BC}$ group $(p<0.001)$ (Figures 3(a) and 3(b)). However, transdermal administration of ZBVO in the PC group was effective in upregulating serum levels of GAS and SP compared to that in the MC group, and the effect of ZBVO was dose-dependent, with the best effect in the HD group. H\&E staining of colon tissue sections showed that no significant morphological damage was observed. Breakage and loss of epithelial cells in the mucosal layer of colonic tissue and reduction of cupped cells were observed in colon tissue sections from rats with STC induced by diphenoxylate tablets. However, ZBVO and mosapride treatment improved the colonic mucosal injury (Figure 4). The levels of relevant inflammatory factors in serum are shown in Figures 5(a) and 5(b). The levels of IL- 6 and TNF- $\alpha$ in the MC group were significantly higher than those in the BC group $(p<0.001)$, and the levels of IL- 6 and TNF- $\alpha$ were reduced after ZBVO and mosapride treatment, and the levels of IL- 6 and TNF- $\alpha$ in ZBVO-HD group were more similar to those in the $\mathrm{BC}$ group. The results showed that $\mathrm{ZBVO}$ had a dose-dependent modulating effect on STC with ZBVO-HD having the best effect.

3.4. Effects of ZBVO on Brain Intestinal Peptide (5-HT and $V I P)$. In order to observe the regulatory effect of $\mathrm{ZBVO}$ on the gut-brain axis in STC rats, the levels of 5-HT and VIP in colon and hippocampal tissues were determined by ELISA. As shown in Figures 6(a) and 6(b), the 5-HT content in the colon and hippocampal tissues of the STC model group was lower than that of the $\mathrm{BC}$ group $(p<0.001)$. Compared with that of the MC group, the 5-HT content in the colon and hippocampal tissues of the ZBVO-LD, ZBVO-MD, ZBVO-HD, and PC groups was significantly 


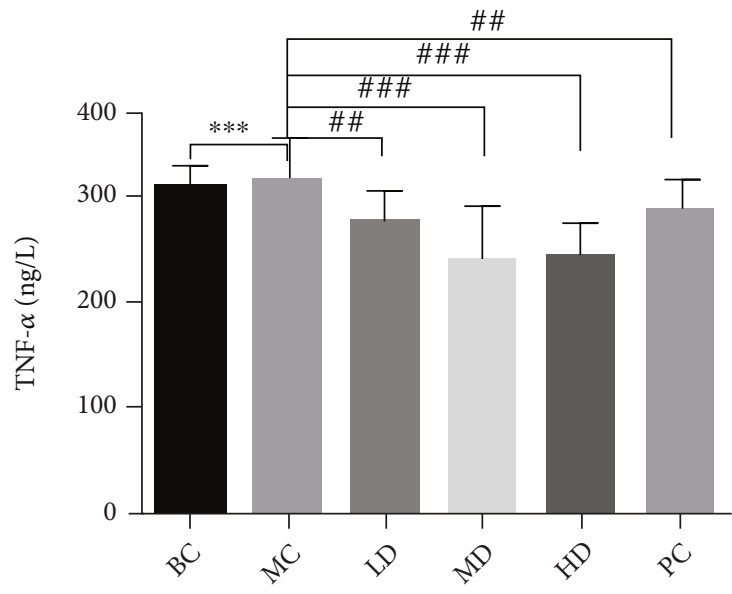

(a)

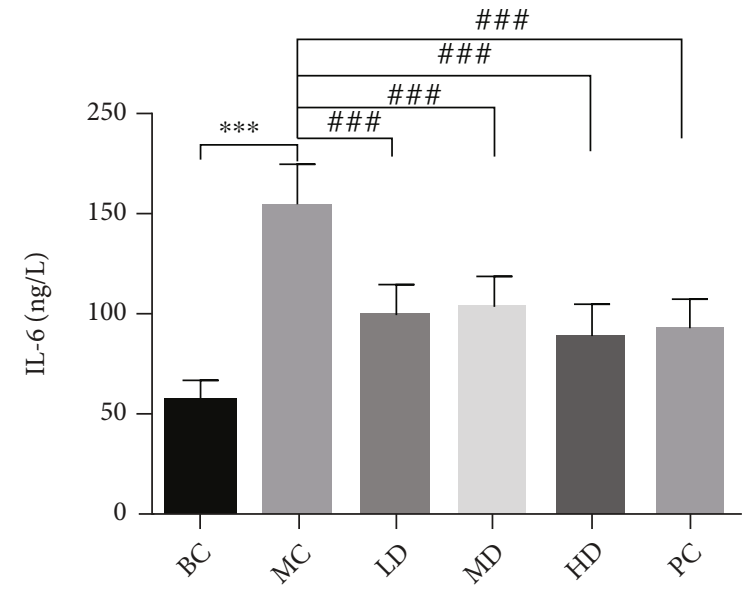

(b)

Figure 5: Effect of ZBVO on the content of IL-6 and TNF- $\alpha$ in the serum $(n=8)$. Values are expressed as mean \pm SEM. ${ }^{*} p<0.05,{ }^{* *} p<0.01$, and ${ }^{* * *} p<0.001$ vs. the BC group. ${ }^{\#} p<0.05,{ }^{\# \#} p<0.01$, and ${ }^{\# \# \#} p<0.001$ vs. the MC group.

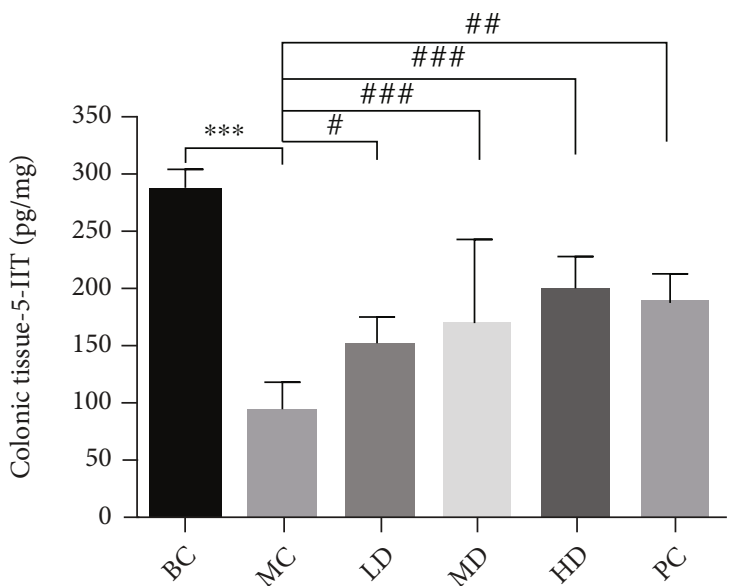

(a)

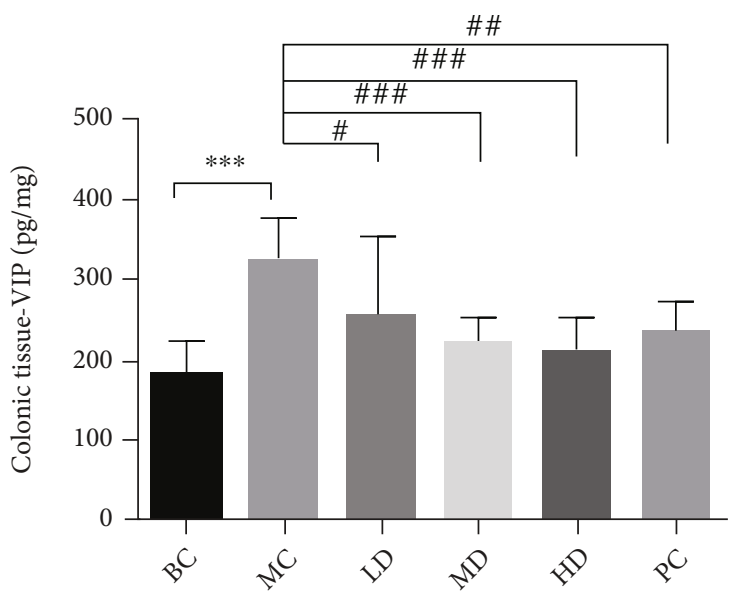

(c)

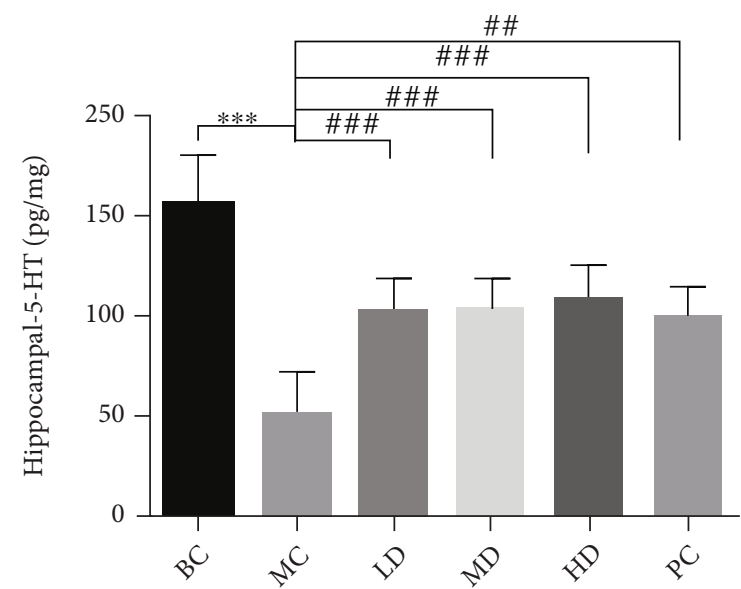

(b)

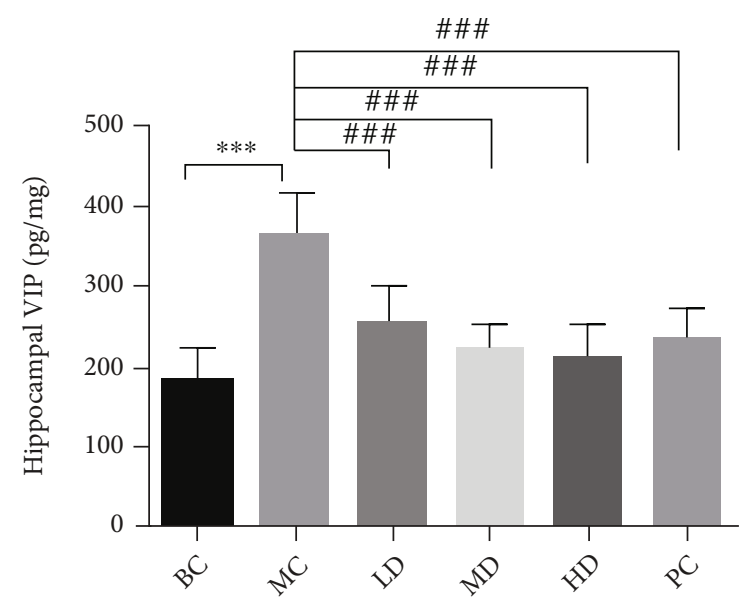

(d)

FIGURE 6: (a-d) Effects of ZBVO treatment on 5-HT and VIP levels in colon and hippocampal tissues $(n=8)$. Values are expressed as mean $\pm \mathrm{SD}, n=8 .{ }^{*} p<0.05,{ }^{* *} p<0.01$, and ${ }^{* * *} p<0.001$ vs. the BC group. ${ }^{\#} p<0.05,{ }^{\# \#} p<0.01$, and ${ }^{\# \# \#} p<0.001$ vs. the MC group. 

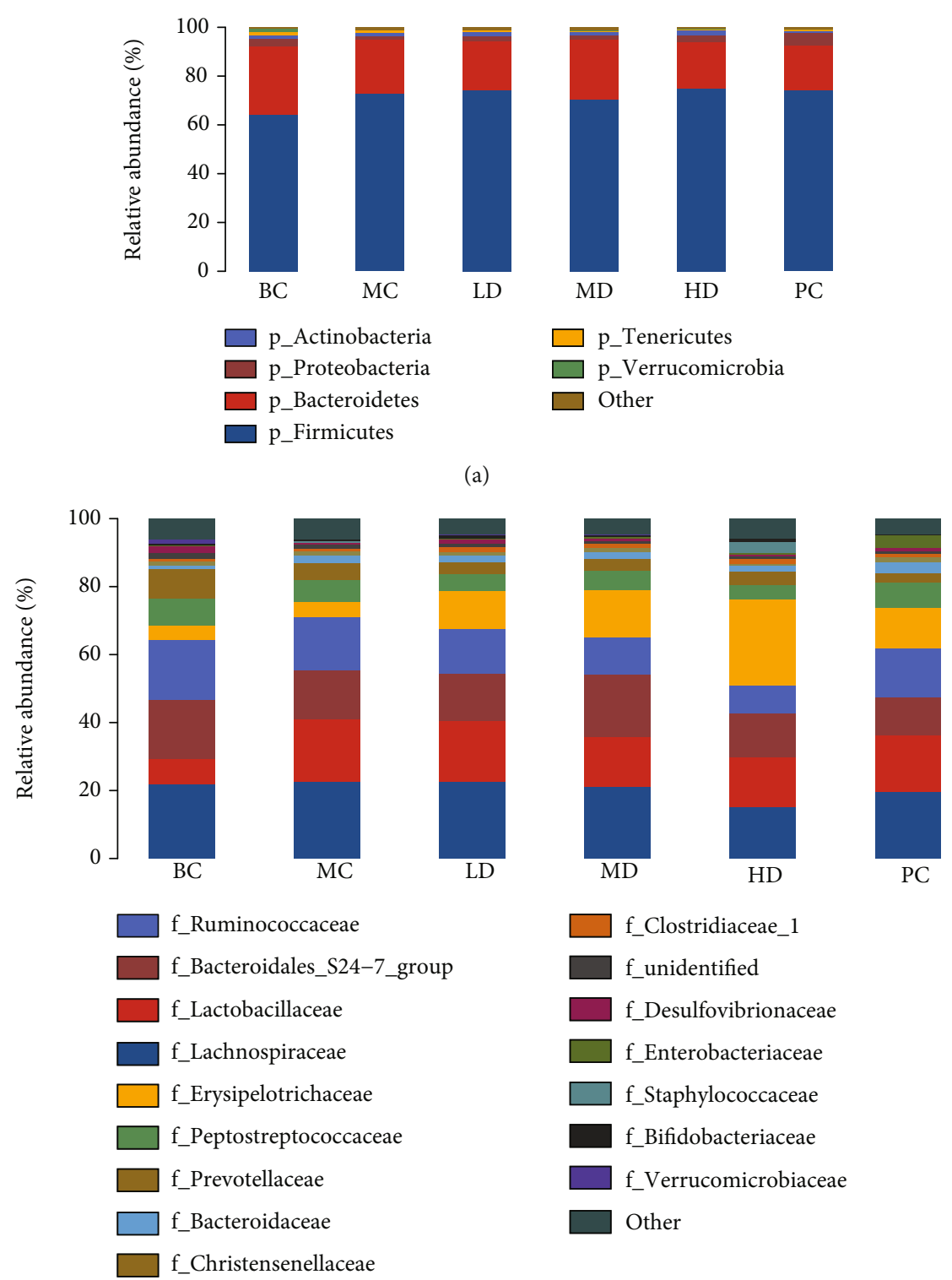

(b)

FIgUre 7: Continued. 


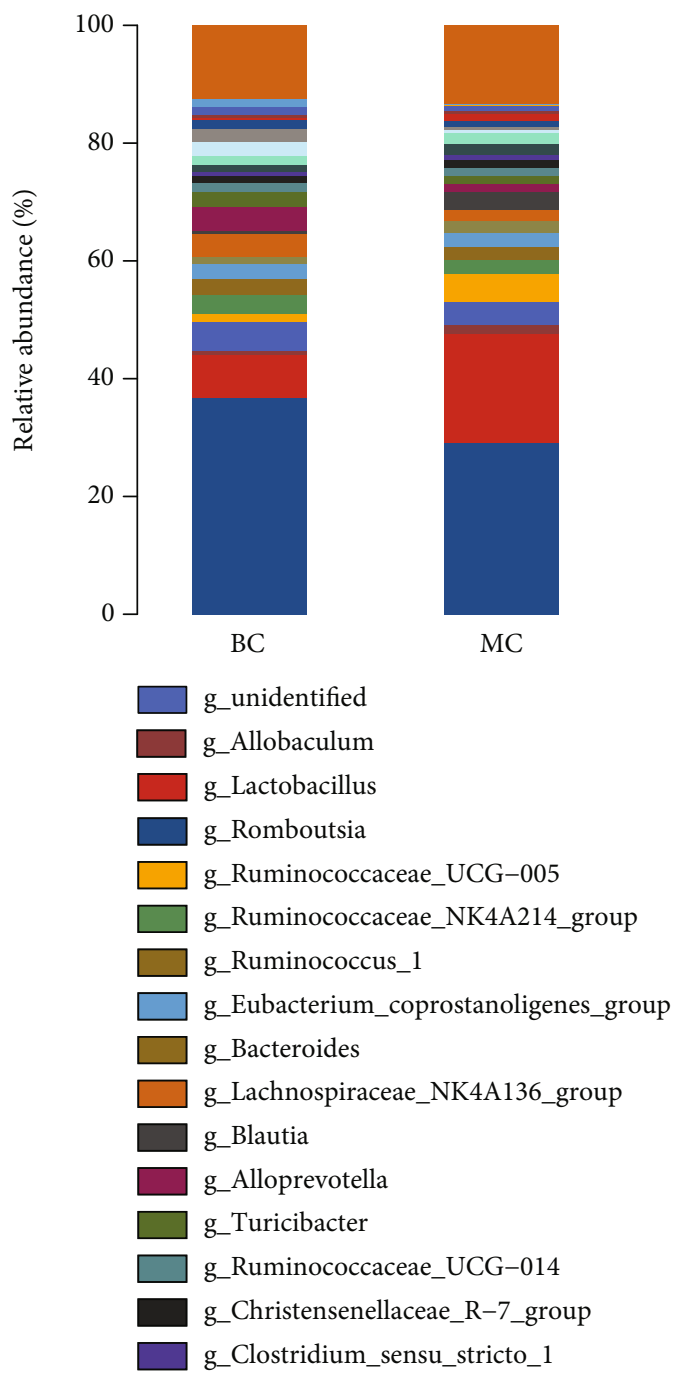

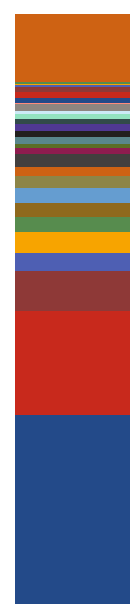

LD

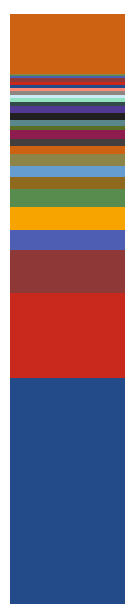

MD

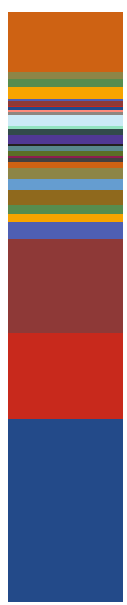

HD

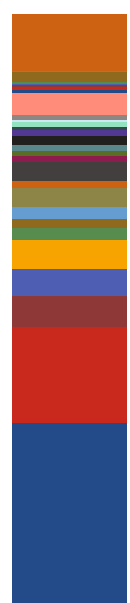

PC

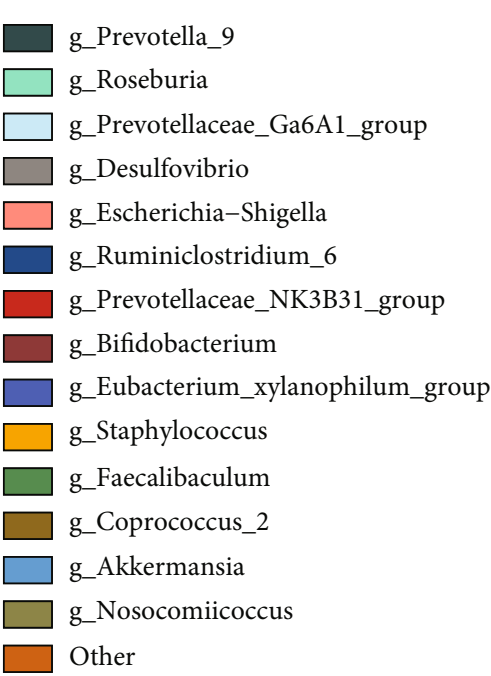

(c)

Figure 7: Analysis of fecal microbial composition of rats in each group: (a) phylum level, (b) family level, and (c) genus level. Less than 1\% of the species were classified as "other" species at all taxonomic levels.

higher $(p<0.05$ or $p<0.01$ or $p<0.001)$, indicating that ZBVO promoted the secretion of 5-HT in the colon and hippocampal tissues. In Figures 6(c) and 6(d), compared with that in the BC group, the VIP content in the colon and hippocampal tissues was decreased in the MC group $(p<0.001)$; compared with the MC group, the ZBVO-LD, ZBVO-MD, ZBVO-HD, and PC groups all decreased the VIP content in the colon and hippocampal tissues to different degrees $(p<0.05$ or $p<0.01$ or $p<0.001)$.

3.5. Regulation of Intestinal Microbiota in DiphenoxylateInduced STC Rats. Thirty-six samples from six groups were evaluated. In order to ensure high coverage for the samples, the data volume of all samples was homogenized to 26,251 sequences. As shown in Supplemental Figure 1A, the rankabundance curve tends to be smoother, indicating the more uniform distribution of species. As shown in Supplemental Figure 1B, the sample curves were all flat and concentrated, indicating that the sequencing data were large enough to reflect the majority of microbial information in the samples. The analysis of the microbiota community structure at the phylum level is shown in Figure $7(\mathrm{a})$, and the results indicate that the identified microorganisms were mainly Firmicutes, Bacteroidetes, Proteobacteria, and Actinobacteria, with Firmicutes accounting for the largest proportion. Compared to the BC group, diphenoxylate-induced STC rats resulted in higher abundance of Firmicutes (72.95\% vs. 64.13\%); Bacteroidetes (22.12\% vs. $28.01 \%)$ and Proteobacteria (1.48\% vs. $3.20 \%)$ were less abundant than those in the $\mathrm{BC}$ group. Compared with the MC group, the abundance of Firmicutes decreased in the MD group and increased in the $\mathrm{LD}, \mathrm{HD}$, and PC groups. The abundance of Bacteroidetes decreased in the LD, HD, and $\mathrm{PC}$ groups but increased in the MD group. The relative abundance of Proteobacteria increased in the LD, MD, HD, and PC groups. The analysis of the microbiota community structure at the family level is shown in Figure 7(b). Diphenoxylate-induced STC rats showed higher family of 


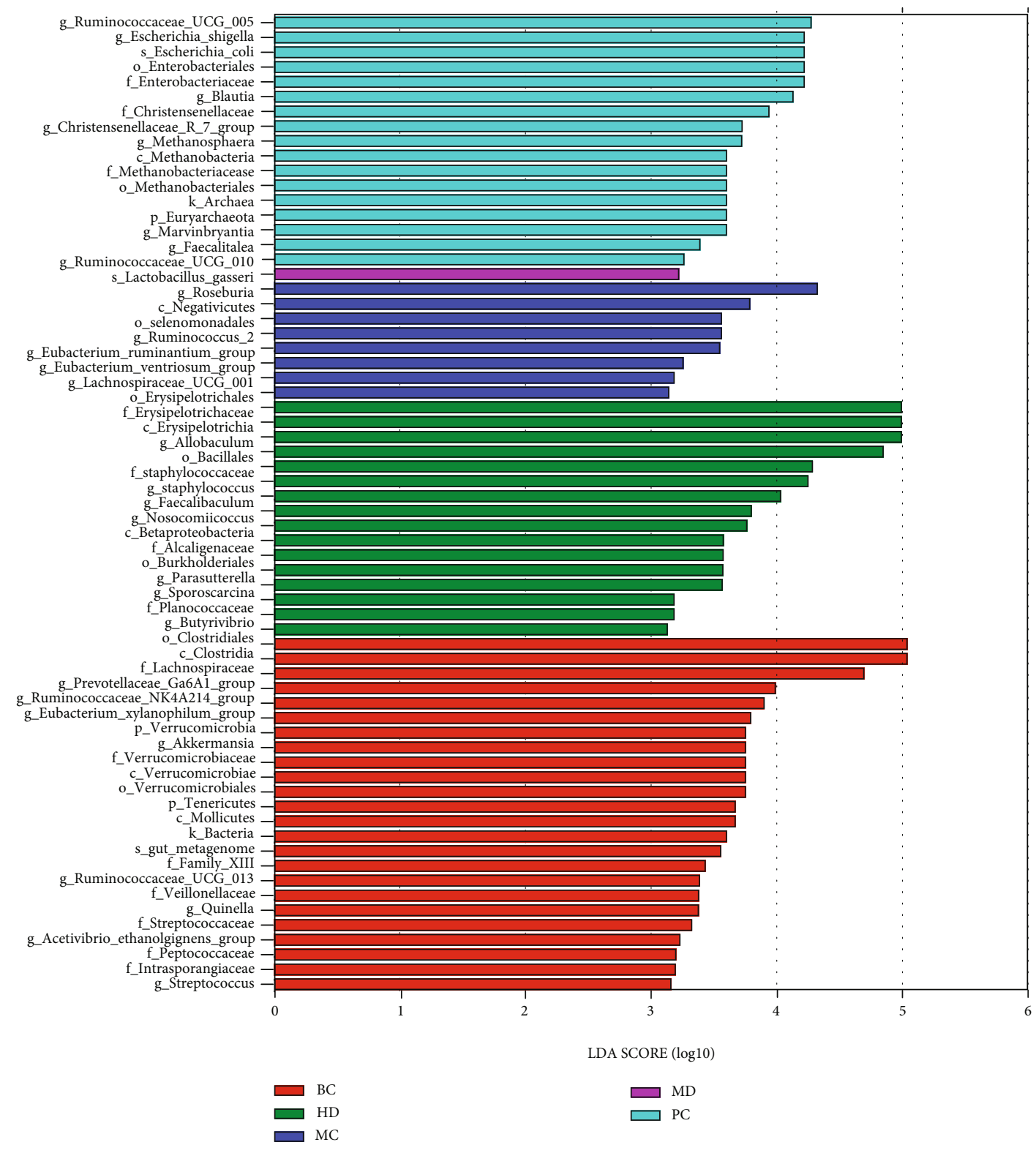

(a)

Figure 8: Continued. 


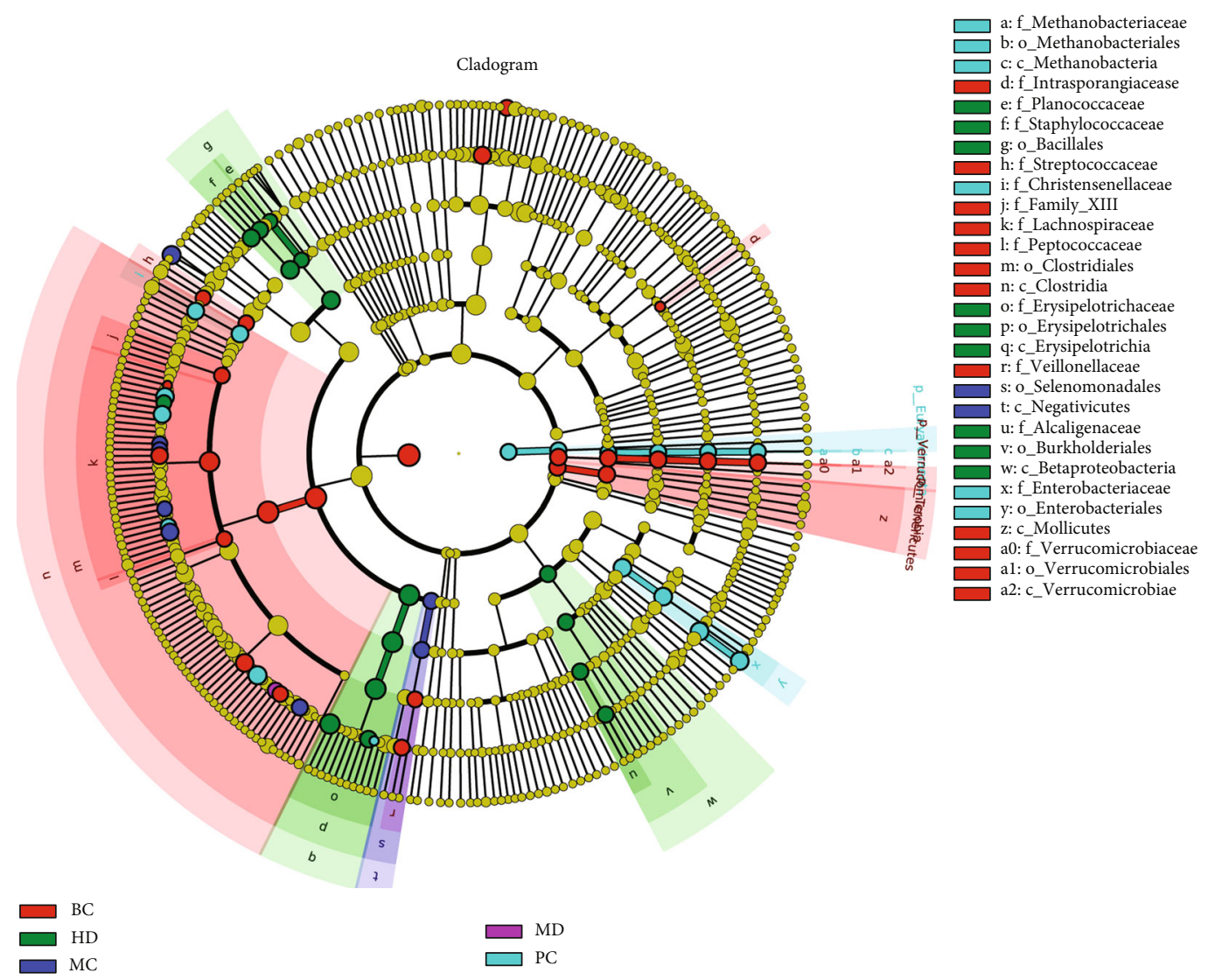

(b)

FIgURE 8: Effect of ZBVO on gut microbiota abundance in STC rats. (a) Distribution histogram based on LDA. (b) Bar plot analysis of community abundance on the genus level.

Ruminococcaceae, Lactobacillaceae, and Erysipelotrichaceae, but lower Lachnospiraceae, Bacteroidales_S24-7_group, Prevotellaceae, and Peptostreptococcaceae families. At the genus level, we found that the relative abundance of Lactobacillus, Ruminococcaceae_UCG-005, and Allobaculum was significantly increased in the STC group compared with the BC group, while the relative abundance of Romboutsia was significantly decreased. Compared with the MC group, the $\mathrm{LD}, \mathrm{MD}, \mathrm{HD}$, and $\mathrm{PC}$ groups increased the relative abundance of Allobaculum, and the $\mathrm{LD}, \mathrm{MD}$, and $\mathrm{HD}$ groups decreased the relative abundance of Romboutsia and increased the relative abundance of Ruminococcaceae_UCG005, while the PC group showed the opposite.

To identify bacterial taxa that differed significantly between groups, LEfSe (linear discriminate analysis size effect) (values $>3$ ) analysis was performed between groups (Figure 8). From phylum to genus, a total of 66 taxa were obtained from all groups. Among them, the BC group was enriched to 24 taxa, the MC group to 8 taxa, the MD group to one taxon, the HD group to 16 taxa, and the PC group to 17 taxa, while the LD group was not enriched to taxa. The results showed that o__Clostridiales, c__Clostridia, and $\mathrm{f}$ Lachnospiraceae were more abundant in the BC group; s_ Lactobacillus_gasseri and g__Roseburia were more abundant in the MC group, while only g_Ruminococcaceae_UCG_ 010 was screened in the $\mathrm{LD}$ group at values $>3$. In the $\mathrm{HD}$ group, the abundance of c_Erysipelotrichia, f_Erysipelotrichaceae, o_Erysipelotrichales, and o__Bacillales was higher, while in the PC group g_Ruminococcaceae_UCG_005, s_ Escherichia_coli, g_Escherichia_Shigella, etc. were more abundant.

The redundancy analysis (RDA) was used to investigate the correlation between the serum levels of host environmental factors IL- 6 and TNF- $\alpha$ and intestinal microbiota in each group of rats. The percentages of the horizontal and vertical coordinates indicate the weight of explanation for the differences in sample composition. The results are shown in Figure 9. Combined RDA and Spearman correlation results show that Lactobacillus and Blautia showed a weak positive correlation to TNF- $\alpha(R>0.3, p<0.05)$, Ruminococcaceae_NK4A214_group showed a negative correlation with IL-6 and TNF- $\alpha(R=-0.39, p<0.05, R=-$ $0.36, p<0.05)$, and Blautia showed a positive correlation with IL-6 $(R=0.37, p<0.05)$; a significant positive correlation was also found between the two environmental factors $(R=0.84, p<0.001)$.

3.6. Regulation of Fecal Metabolomics in DiphenoxylateInduced STC Rats. The positive and negative ion metabolic profiles of each group of stool samples were analyzed by UPLC-Q-TOF-MS; these results were combined and analyzed to establish orthogonal partial least squares 


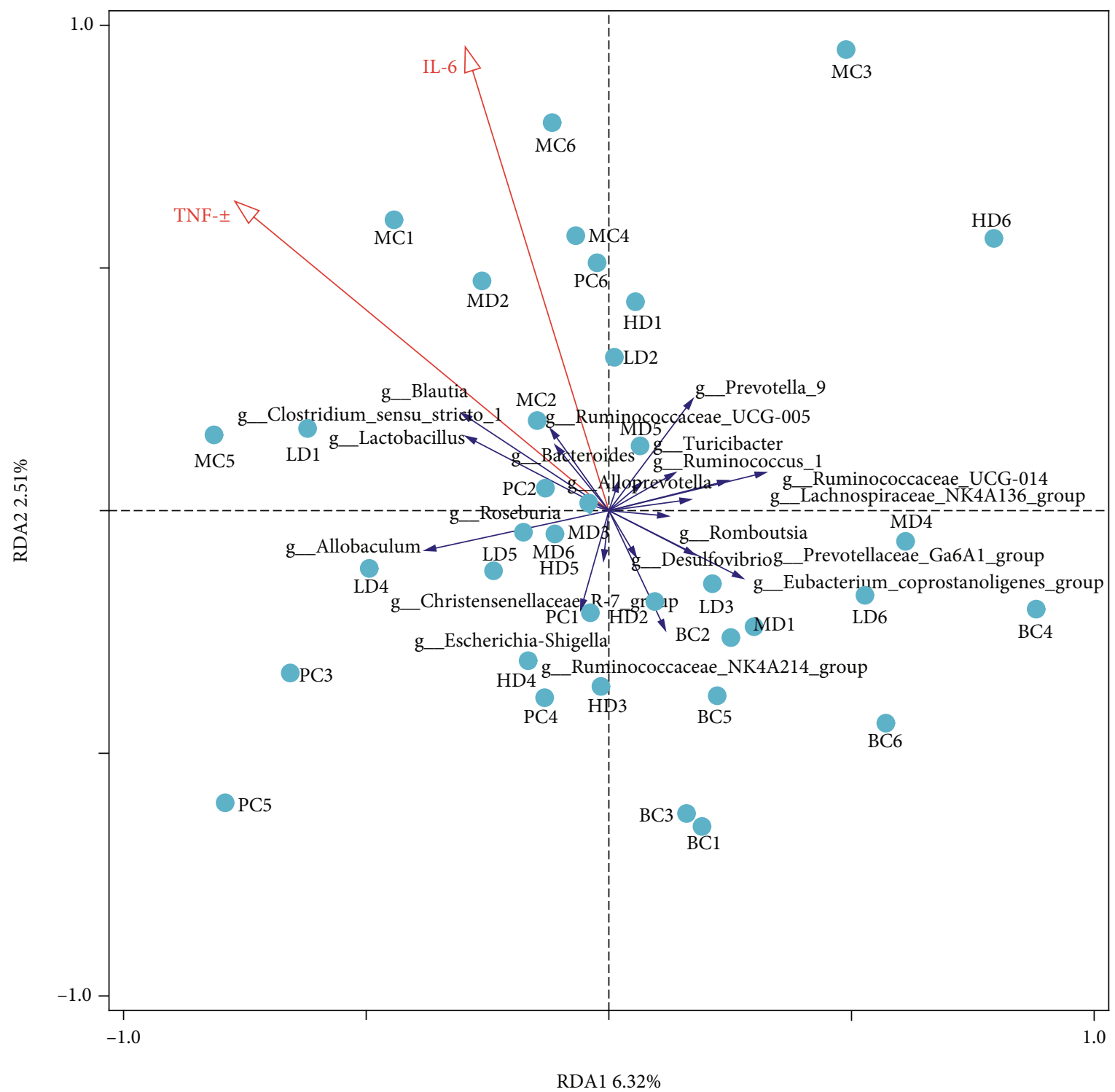

Figure 9: Analysis of RDA correlation between inflammatory factors and intestinal microorganism genus level where the dots represent the 36 samples $(n=6)$. Red rays represent the two environmental factors, IL- 6 and TNF- $\alpha$, and blue rays represent different species. The length of the rays represents the degree of their influence on the sample communities, and the direction of the rays represents the direction of increasing abundance. If the angle between the two rays is acute, it means there is a positive correlation; if it is obtuse, then there is a negative correlation; and if it is right angle, there is no correlation.

discrimination analysis (OPLS-DA) (Figure 10). The separation between the MC and BC groups was clearly shown in both OPLS-DA analyses (Figure 10(a)), indicating that the metabolic phenotype of STC rats has changed significantly. The fecal metabolic profile of the LD, MD, and HD groups, with the HD group being the most obvious, changed similarly to the BC group and showed partial overlap, indicating that the high dose of ZBVO had a significant ameliorating effect on STC rats. In addition, in the OPLS-DA model validation, the model parameters $R^{2} Y=0.927$ and $Q^{2}=0.635$, $p<0.005$, indicate that the model has good adaptability (Figure 10(b)). OPLS-DA analysis provides better separation and can be used to further search for biomarkers (Figures 10(c)-10(f)).
The HMDB online database and KEGG database were used to screen metabolites using $p<0.05$ and VIP $\geq 1$ parameters. Thirty significantly changed metabolites were identified in feces (Table 2), 25 were significantly lower in the $\mathrm{MC}$ group than in the $\mathrm{BC}$ group, and five were significantly upregulated in the STC rats. After ZBVO treatment, some metabolites did not show significant changes in the diphenoxylate tablet-induced model group compared to the treatment group. However, uridine, glycochenodeoxycholate, arachidonic acid (peroxide-free), quinolinate, 1methylnicotinamide, xanthosine, 3-phenylpropanoic acid, azelaic acid, L-malic acid, and deoxyadenosine showed a significant inverse trend (Table 3). This suggests that the mechanism of action of ZBVO in regulating STC may be related 

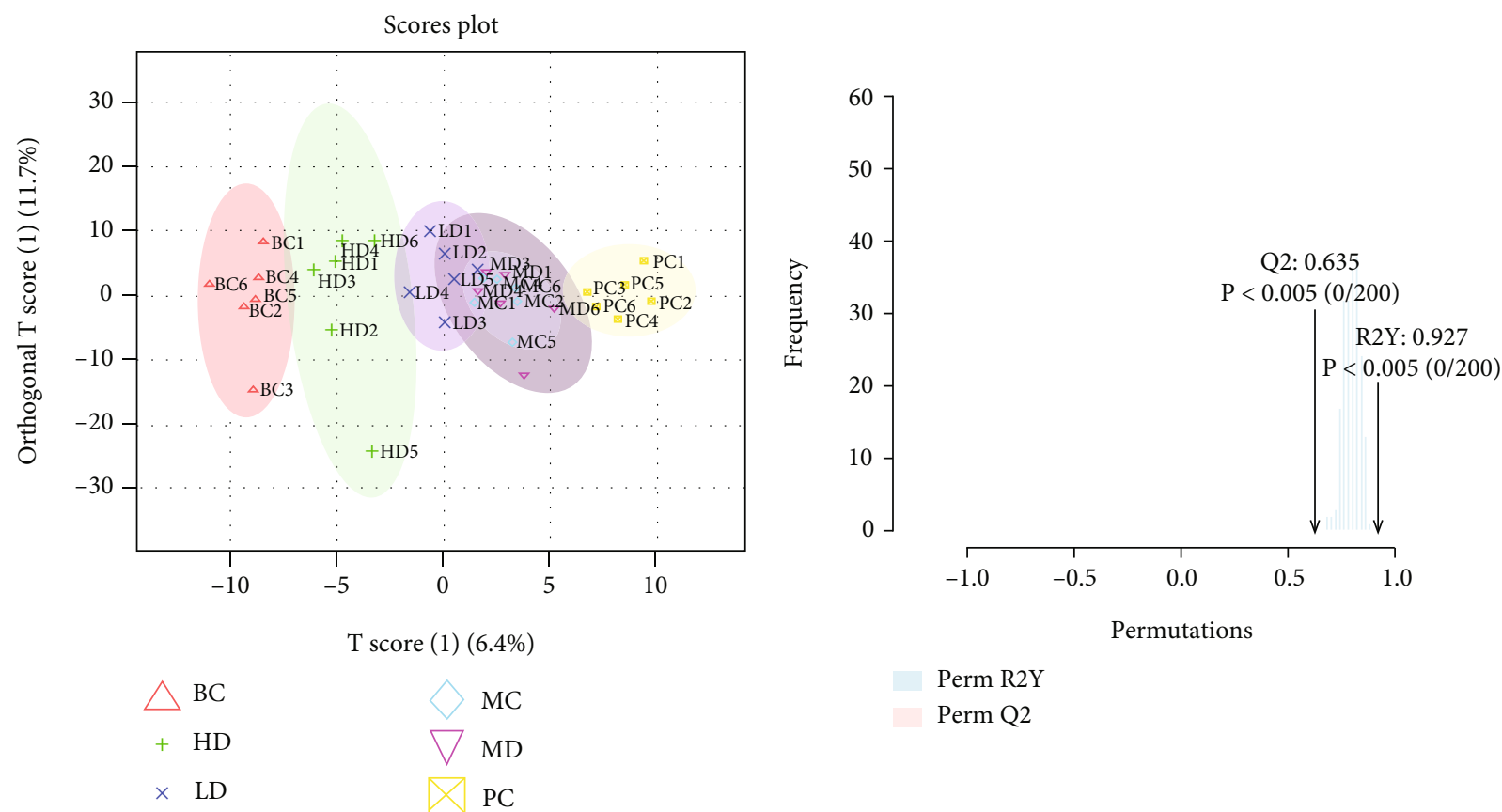

(a)

Perm R2Y

Perm Q2

(b)
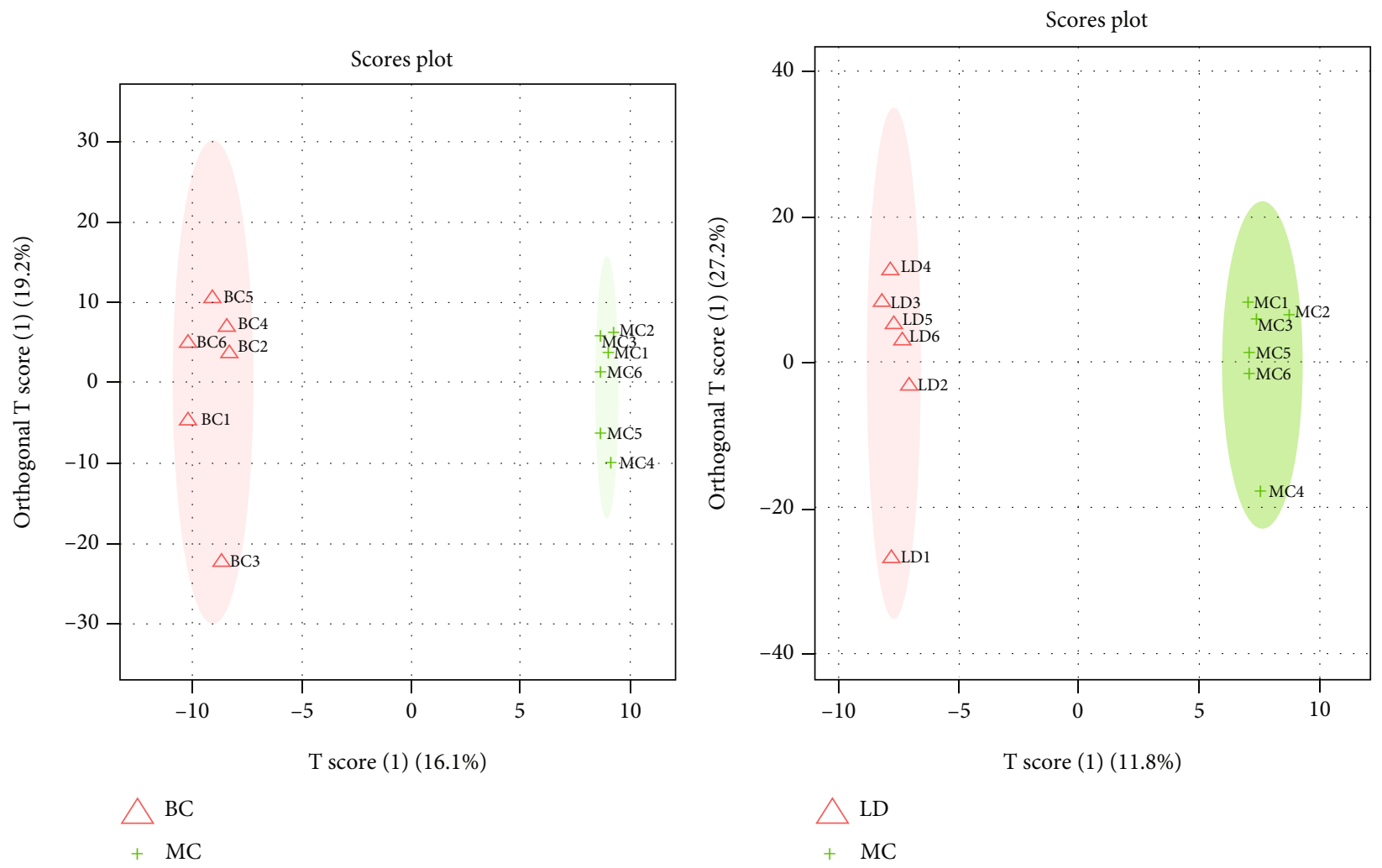

(c)

(d)

Figure 10: Continued. 


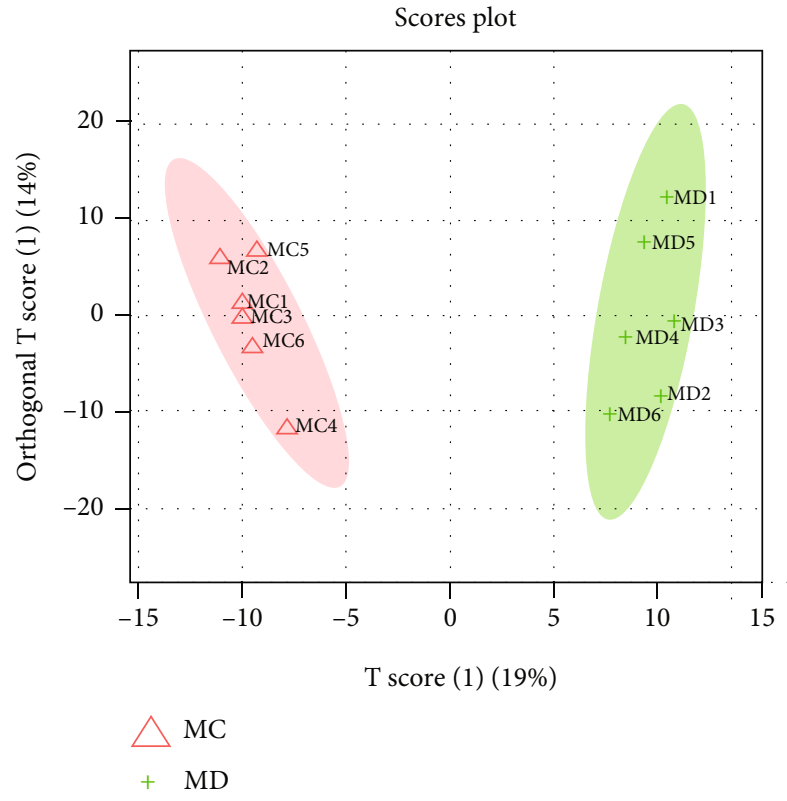

(e)

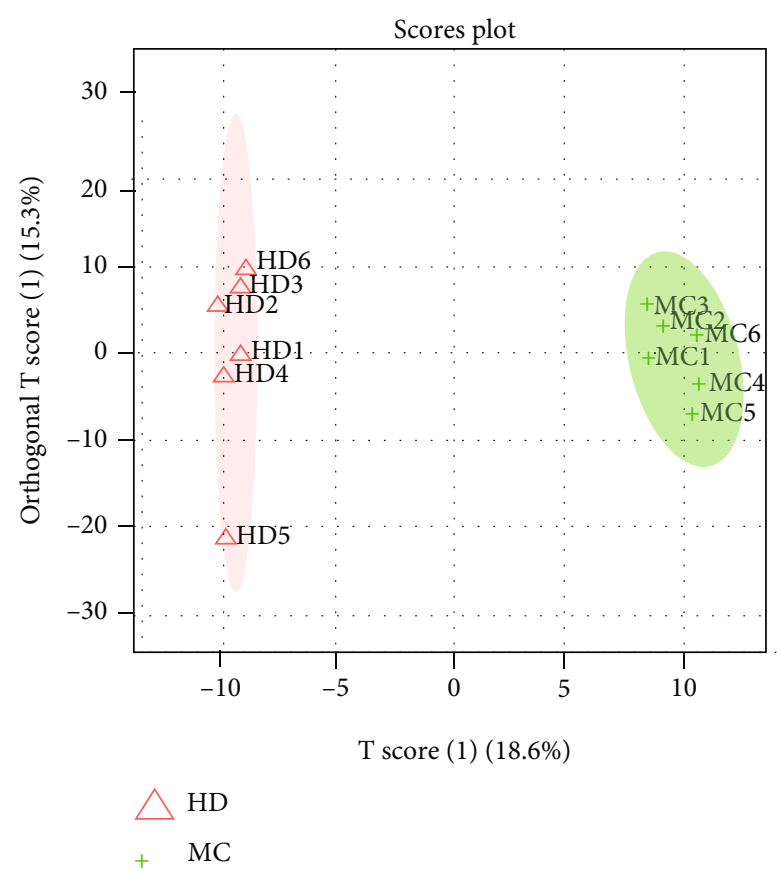

(f)

FIGURE 10: Supervised discriminant analysis of fecal metabolome in STC rats $(n=6)$. (a) OPLS-DA analysis and (b) validation of six groups. OPLS-DA analysis of (c) BC vs. MC, (d) LD vs. MC, (e) MD vs. MC, and (f) HD vs. MC.

to these metabolites, likely by interfering with different metabolic pathways.

To investigate the potential metabolic pathways of ZBVO in STC, the 10 metabolites with significant differences in Table 3 were subjected to metabolic pathway analysis and the results showed that $\mathrm{ZBVO}$ regulates seven metabolic pathways in STC rats (Figure 11), namely, nicotinate and nicotinamide metabolism, purine metabolism, citrate cycle (TCA cycle), pyruvate metabolism, arachidonic acid metabolism, pyrimidine metabolism, and primary bile acid biosynthesis.

\subsection{Relevance Analysis between Gut Microbiota and Fecal} Biomarkers. Pearson correlation analysis was used to explore the potential association between gut microbiota genera and fecal biomarkers. The correlation between the two is presented the heat map plot in Figure 12. The results showed that g_Allobaculum and g_Faecalibaculum were negatively correlated with arachidonic acid (peroxide-free), g Sporosarcina was negatively correlated with glycochenodeoxycholate, and g__Nosocomiicoccus and g_Staphylococcus were positively correlated with xanthosine.

\section{Discussion}

STC is a common form of functional constipation, characterized by slow colonic motility and delayed elimination of feces [23]. TCM has been used to prevent and treat various diseases for thousands of years, which is characterized by multicomponents, multitargets, personalized, and holistic therapeutic strategies $[24,25]$. ZS and BZ are two classical aromatic herbs commonly prescribed for laxative purposes, and most studies have focused on the efficacy of oral administration, while the role of ZBVO in modulating STC and its mechanism are still unclear. In this study, the effect of ZBVO in regulating STC and its mechanism were investigated by transdermal administration at the Shenque acupoint. 16S rRNA gene sequencing and fecal metabolomics were also used to elucidate the mechanism of action of ZBVO in laxatives. Our study showed that ZBVO has therapeutic effects in STC rats, and the main mechanisms are anti-inflammatory, regulation of gastrointestinal hormones, regulation of brain and intestinal peptides, and regulation of intestinal microbiota and endogenous metabolites. ZBVO improved the typical symptoms of STC induced by compound diphenoxylate tablets, especially in the MD and HD groups.

It is hypothesized that the intestinal motility-promoting effect of ZBVO is related to its chemical composition and activity. The highest levels of the chemical components Dlimonene, beta-myrcene, and gamma-terpinene found in Citrus aurantium volatile oil are associated with the treatment of STC. D-Limonene causes excitation of the smooth muscle in the gastrointestinal tract and regulates immunity [26]. It also has anti-inflammatory and antiseptic properties and can reduce gastric $\mathrm{pH}$ and increase the effect of gastric mucus [27, 28]. Zheng et al. [29] found that D-limonene significantly promoted gastric emptying and intestinal peristalsis and inhibited gastrointestinal smooth muscle contraction. The volatile oil of $\mathrm{ZS}$ is predicted to exert its pharmacological effects mainly through acetylcholinesterase (ACHE), prostaglandin-endoperoxide synthase 2 (PTGS2), norepinephrine transporter gene (SLC6A2), peroxisome proliferator-activated receptor (PPARA), cholinergic 
TABLe 2: Potential biomarkers in rat feces after modeling $(n=6)$.

\begin{tabular}{|c|c|c|c|c|c|c|}
\hline Name & Formula & Detected $\mathrm{m} / \mathrm{z}$ & VIP value & $\mathrm{MC} / \mathrm{BC}$ & HMDB ID & KEGG \\
\hline Protoporphyrin IX & $\mathrm{C}_{34} \mathrm{H}_{34} \mathrm{~N}_{4} \mathrm{O}_{4}$ & 563.2598 & 1.49 & $\downarrow^{\#}$ & HMDB0000241 & C02191 \\
\hline Uridine & $\mathrm{C}_{9} \mathrm{H}_{12} \mathrm{~N}_{2} \mathrm{O}_{6}$ & 243.0648 & 2.09 & $\downarrow^{\# \#}$ & HMDB0000296 & C00299 \\
\hline Nervonic acid & $\mathrm{C}_{24} \mathrm{H}_{46} \mathrm{O}_{2}$ & 365.3481 & 1.63 & $\downarrow^{\#}$ & HMDB0002368 & C08323 \\
\hline Cer(d18:1/18:1(9Z)) & $\mathrm{C}_{36} \mathrm{H}_{69} \mathrm{NO}_{3}$ & 564.5288 & 1.83 & $\downarrow^{\# \#}$ & HMDB0004948 & C00195 \\
\hline Lathosterol & $\mathrm{C}_{27} \mathrm{H}_{46} \mathrm{O}$ & 369.3463 & 1.43 & $\downarrow^{\#}$ & HMDB0001170 & C01189 \\
\hline Lanosterol & $\mathrm{C}_{30} \mathrm{H}_{50} \mathrm{O}$ & 409.3744 & 1.75 & $\downarrow^{\#}$ & HMDB0001251 & $\mathrm{C} 01724$ \\
\hline Myristoleic acid & $\mathrm{C}_{14} \mathrm{H}_{26} \mathrm{O}_{2}$ & 191.1767 & 1.70 & $\downarrow^{\#}$ & HMDB0002000 & $\mathrm{C} 08322$ \\
\hline N-Glycolylneuraminic acid & $\mathrm{C}_{11} \mathrm{H}_{19} \mathrm{NO}_{10}$ & 326.1047 & 1.46 & $\downarrow^{\#}$ & HMDB0000833 & $\mathrm{C} 03410$ \\
\hline Glycochenodeoxycholate & $\mathrm{C}_{26} \mathrm{H}_{43} \mathrm{NO}_{5}$ & 432.3012 & 1.83 & $\downarrow^{\# \#}$ & HMDB0000637 & C05466 \\
\hline Arachidonic acid (peroxide-free) & $\mathrm{C}_{20} \mathrm{H}_{32} \mathrm{O}_{2}$ & 269.2062 & 1.85 & $\downarrow^{\# \#}$ & HMDB0001043 & C00219 \\
\hline Phytanic acid & $\mathrm{C}_{20} \mathrm{H}_{40} \mathrm{O}_{2}$ & 354.3308 & 1.52 & $\downarrow^{\#}$ & HMDB0000801 & C01607 \\
\hline L-Glutamine & $\mathrm{C}_{5} \mathrm{H}_{10} \mathrm{~N}_{2} \mathrm{O}_{3}$ & 145.0521 & 1.63 & $\downarrow^{\#}$ & HMDB0000641 & C00064 \\
\hline Uracil & $\mathrm{C}_{4} \mathrm{H}_{6} \mathrm{~N}_{2} \mathrm{O}_{2}$ & 113.0326 & 1.51 & $\downarrow^{\#}$ & HMDB0000300 & C00106 \\
\hline Quinolinate & $\mathrm{C}_{7} \mathrm{H}_{5} \mathrm{NO}_{4}$ & 166.0165 & 1.62 & $\downarrow^{\#}$ & HMDB0000232 & C03722 \\
\hline Alpha-D-glucose & $\mathrm{C}_{6} \mathrm{H}_{12} \mathrm{O}_{6}$ & 179.0571 & 1.57 & $\downarrow^{\#}$ & HMDB0003345 & C00267 \\
\hline L-Serine & $\mathrm{C}_{3} \mathrm{H}_{7} \mathrm{NO}_{3}$ & 104.0343 & 1.44 & $\downarrow^{\#}$ & HMDB0000187 & C00065 \\
\hline 1,7-Dimethylxanthine & $\mathrm{C}_{7} \mathrm{H}_{8} \mathrm{~N}_{4} \mathrm{O}_{2}$ & 180.0554 & 1.84 & $\downarrow^{\# \#}$ & HMDB0001860 & C13747 \\
\hline D-Threitol & $\mathrm{C}_{4} \mathrm{H}_{10} \mathrm{O}_{4}$ & 143.0353 & 1.63 & $\downarrow^{\#}$ & HMDB0004136 & C16884 \\
\hline trans-Dehydroandrosterone & $\mathrm{C}_{19} \mathrm{H}_{28} \mathrm{O}_{2}$ & 287.1868 & 1.56 & $\downarrow^{\#}$ & HMDB0000077 & C01227 \\
\hline 1-Methylnicotinamide & $\mathrm{C}_{7} \mathrm{H}_{9} \mathrm{~N}_{2} \mathrm{O}$ & 138.0576 & 2.16 & $\downarrow^{\# \#}$ & HMDB0000699 & C02918 \\
\hline L-Asparagine & $\mathrm{C}_{4} \mathrm{H}_{8} \mathrm{~N}_{2} \mathrm{O}_{3}$ & 113.0240 & 1.51 & $\downarrow^{\#}$ & HMDB0000168 & C00152 \\
\hline Xanthosine & $\mathrm{C}_{10} \mathrm{H}_{12} \mathrm{~N}_{4} \mathrm{O}_{6}$ & 285.0836 & 1.98 & $\downarrow^{\# \#}$ & HMDB0000299 & $\mathrm{C} 01762$ \\
\hline D-Sorbitol & $\mathrm{C}_{6} \mathrm{H}_{14} \mathrm{O}_{6}$ & 163.0621 & 1.44 & $\downarrow^{\#}$ & HMDB0000247 & C00794 \\
\hline 4-Hydroxycinnamic acid & $\mathrm{C}_{9} \mathrm{H}_{8} \mathrm{O}_{3}$ & 147.0415 & 1.51 & $\downarrow^{\#}$ & HMDB0002035 & C00811 \\
\hline L-Malic acid & $\mathrm{C}_{4} \mathrm{H}_{6} \mathrm{O}_{5}$ & 133.0146 & 1.52 & $\downarrow^{\#}$ & HMDB0000156 & C00149 \\
\hline 3-Phenylpropanoic acid & $\mathrm{C}_{9} \mathrm{H}_{10} \mathrm{O}_{2}$ & 149.0622 & 1.50 & $\uparrow^{\#}$ & HMDB0000764 & C05629 \\
\hline Aminopterin & $\mathrm{C}_{19} \mathrm{H}_{20} \mathrm{~N}_{8} \mathrm{O}_{5}$ & 439.1542 & 1.95 & $\uparrow^{\# \#}$ & HMDB0001833 & D02527 \\
\hline Azelaic acid & $\mathrm{C}_{9} \mathrm{H}_{16} \mathrm{O}_{4}$ & 187.0997 & 2.05 & $\uparrow^{\# \#}$ & HMDB0000784 & C08261 \\
\hline Deoxyadenosine & $\mathrm{C}_{10} \mathrm{H}_{13} \mathrm{~N}_{5} \mathrm{O}_{3}$ & 252.1042 & 1.62 & $\uparrow^{\#}$ & HMDB0000101 & C00559 \\
\hline 1-Methylhistamine & $\mathrm{C}_{6} \mathrm{H}_{11} \mathrm{~N}_{3}$ & 112.0843 & 1.64 & $\uparrow^{\#}$ & HMDB0000898 & $\mathrm{C} 05127$ \\
\hline
\end{tabular}

MC/BC: comparison between the model control group and the blank control group, ${ }^{\#} p<0.05$ and ${ }^{\# \#} p<0.01$; " $\downarrow$ " or " $\uparrow$ " compared with the blank control group, the metabolites in the model control group decreased or increased significantly.

TABLe 3: Differential metabolites in rat feces after administration $(n=6)$.

\begin{tabular}{|c|c|c|c|c|c|c|}
\hline Name & Formula & Detected $\mathrm{m} / \mathrm{z}$ & VIP value $(\mathrm{MD} / \mathrm{MC})$ & VIP value (HD/MC) & HMDB ID & KEGG \\
\hline Uridine & $\mathrm{C}_{9} \mathrm{H}_{12} \mathrm{~N}_{2} \mathrm{O}_{6}$ & 243.0648 & $1.54 \uparrow^{\#}$ & - & HMDB0000296 & C00299 \\
\hline Glycochenodeoxycholate & $\mathrm{C}_{26} \mathrm{H}_{43} \mathrm{NO}_{5}$ & 432.3012 & $1.47 \uparrow^{\#}$ & - & HMDB0000637 & $\mathrm{C} 05466$ \\
\hline Arachidonic acid (peroxide-free) & $\mathrm{C}_{20} \mathrm{H}_{32} \mathrm{O}_{2}$ & 269.2062 & $1.54 \uparrow^{\#}$ & - & HMDB0001043 & C00219 \\
\hline Quinolinate & $\mathrm{C}_{7} \mathrm{H}_{5} \mathrm{NO}_{4}$ & 166.0165 & $1.69 \uparrow^{\# \#}$ & $1.75 \uparrow_{\# \#}$ & HMDB0000232 & $\mathrm{C} 03722$ \\
\hline 1-Methylnicotinamide & $\mathrm{C}_{7} \mathrm{H}_{9} \mathrm{~N}_{2} \mathrm{O}$ & 138.0576 & $1.45 \uparrow^{\#}$ & - & HMDB0000699 & C02918 \\
\hline Xanthosine & $\mathrm{C}_{10} \mathrm{H}_{12} \mathrm{~N}_{4} \mathrm{O}_{6}$ & 285.0836 & $1.48 \uparrow^{\#}$ & $1.91 \uparrow_{\# \#}$ & HMDB0000299 & C01762 \\
\hline 3-Phenylpropanoic acid & $\mathrm{C}_{9} \mathrm{H}_{10} \mathrm{O}_{2}$ & 149.0622 & $1.81 \downarrow^{\# \#}$ & - & HMDB0000764 & C05629 \\
\hline Azelaic acid & $\mathrm{C}_{9} \mathrm{H}_{16} \mathrm{O}_{4}$ & 187.0997 & $1.78 \downarrow^{\# \#}$ & - & HMDB0000784 & C08261 \\
\hline L-Malic acid & $\mathrm{C}_{4} \mathrm{H}_{6} \mathrm{O}_{5}$ & 133.0146 & - & $1.79 \uparrow^{\# \#}$ & HMDB0000156 & C00149 \\
\hline Deoxyadenosine & $\mathrm{C}_{10} \mathrm{H}_{13} \mathrm{~N}_{5} \mathrm{O}_{3}$ & 252.1042 & - & $1.48 \downarrow^{\#}$ & HMDB0000101 & C00559 \\
\hline
\end{tabular}

Note: " $\downarrow$ " or " " compared with the model control group, the metabolites in the middle-dose (MD) group and the high-dose (HD) group decreased or increased significantly. 
Nicotinate and nicotinamide metabolism

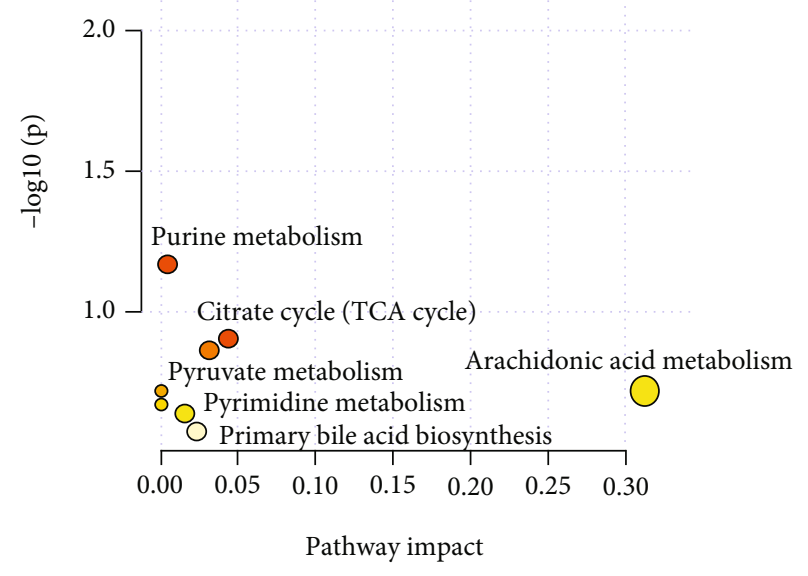

FIGURE 11: Summary of pathway analysis with MetPA.

muscarinic 2 (CHRM2), and other related target proteins. It is mainly related to neuroactive ligand-receptor interaction, endocrine resistance, serotonergic synapse, cyclic adenosine monophosphate (cAMP), calcium signaling pathway, and so on [30]. To a certain extent, it confirms the reliability of the ZBVO study for the treatment of STC. Here, the chemical composition of ZBVO was analyzed by GC-MS, and 38 chemical components were identified, among which $\mathrm{D}$ limonene (42.72\%), atractylon (27.51\%), and $\gamma$-elemene (7.83\%) were identified as the three most abundant chemical components.

Gastrointestinal hormones regulate the movement, secretion, and absorption of the digestive system through different signaling pathways [31]. GAS is mainly secreted by the mucosa of the gastric sinus, duodenum, and small intestine [32]. SP is an endogenous peptide involved in immune regulation and cell proliferation, which has a facilitative effect in the contraction of intestinal smooth muscle and promotes gastrointestinal motility by stimulating the secretion of water and electrolytes from the small intestine and colonic mucosa. Additionally, it regulates the inflammatory response [33]. Some studies have shown that GAS and SP levels were significantly lower in patients with constipation [34]. In this study, the ZBVO administration and PC groups were able to increase the levels of GAS and SP in a dose-dependent manner. Therefore, ZBVO has a laxative effect and promotes intestinal motility after transdermal administration.

Current research in the field suggests that the pathogenesis of STC is closely related to disorders of the brain-gut axis [35]. 5-HT and VIP are expressed in both colonic and hippocampal tissues of animals [36]. 5-HT activates afferent nerve fibers in the intestinal mucosa to regulate local excitation or inhibition, thereby promoting colonic motility and transmission [37]. Bassotti et al. [38] found that the level of 5-HT 4 receptors in STC patients showed a positive correlation with colonic transport capacity, suggesting that the transport system of the colon is closely related to 5-HT. 5-
HT is a brain intestinal peptide that binds to the 5hydroxytryptamine transporter receptor (SERT) and acts on gastrointestinal motility, and some studies have reported that dysbiosis of the intestinal microbiota can have an effect on the expression level of SERT [39]. Clostridium prazmowski and Escherichia coli secretion products can exert a constraining effect on the synthesis rate of 5-HT, thus affecting the movement of smooth muscle in the gastrointestinal tract [40]. VIP is composed of 28 amino acids, which can relax the smooth muscle of the gastrointestinal tract, thereby slowing down intestinal peristalsis and intestinal dynamics, making defecation difficult and eventually causing constipation. Clinical trials indicate that patients with constipation have lower levels of VIP expression than normal [41]. In the present study, we found that ZBVO could upregulate 5-HT in colonic and hippocampal tissues, while downregulating VIP expression, together exerting a regulatory effect on STC.

Dysbiosis of the intestinal microbiota is closely associated with the development of several pathogenic conditions, such as obesity, gastrointestinal disorders, diabetes, and Alzheimer's disease $[42,43]$. In the present study, highthroughput sequencing of the 16S rRNA gene V4 region of the intestinal microbiota showed that the intestinal microbiota of rats induced by diphenoxylate tablets differed significantly from that of normal rats in terms of diversity and composition. And the abundance of Firmicutes was increased in the intestine of rats induced by compound diphenoxylate tablets compared with the $\mathrm{BC}$ group, but the abundance of the Bacteroidetes and Proteobacteria was lower than that of the $\mathrm{BC}$ group, and the community composition was changed by reducing Romboutsia bacteria as well as increasing Proteobacteria, Allobaculum, and Ruminococcaceae after ZBVO administration. To further understand how volatile oils improve constipation in SD rats, the genus level was explored at the level of in vivo microbiota. IL- 6 and TNF- $\alpha$ are closely related to the development of STC [21]. The present study found that ZBVO significantly reduced the serum levels of IL- 6 and TNF- $\alpha$ and moderately slowed down the inflammatory response. Moreover, Lactobacillus and Blautia showed a weak positive correlation with TNF$\alpha$, Ruminococcaceae showed a negative correlation with IL6 and TNF- $\alpha$, and Blautia showed a positive correlation with IL-6. There was also a significant positive correlation found between IL- 6 and TNF- $\alpha$, suggesting that the change of intestinal microbiota is involved in the regulation of inflammatory factor expression, and the joint action of both contributes to the development and treatment of STC.

Fecal metabolomics indicates that the MC group is clearly separated from the $\mathrm{BC}$ group, while the HD group was close to the $\mathrm{BC}$ group and showed partial overlap, indicating that metabolite levels in vivo had changed significantly compared with the $\mathrm{BC}$ group, and after administration of ZBVO, there was a tendency to return to normal performance levels. A total of 30 potential biomarkers associated with STC were identified, and ZBVO intervention significantly regressed 10 of them. Metabolic pathway enrichment analysis revealed that ZBVO may regulate metabolic pathways such as nicotinate and nicotinamide 


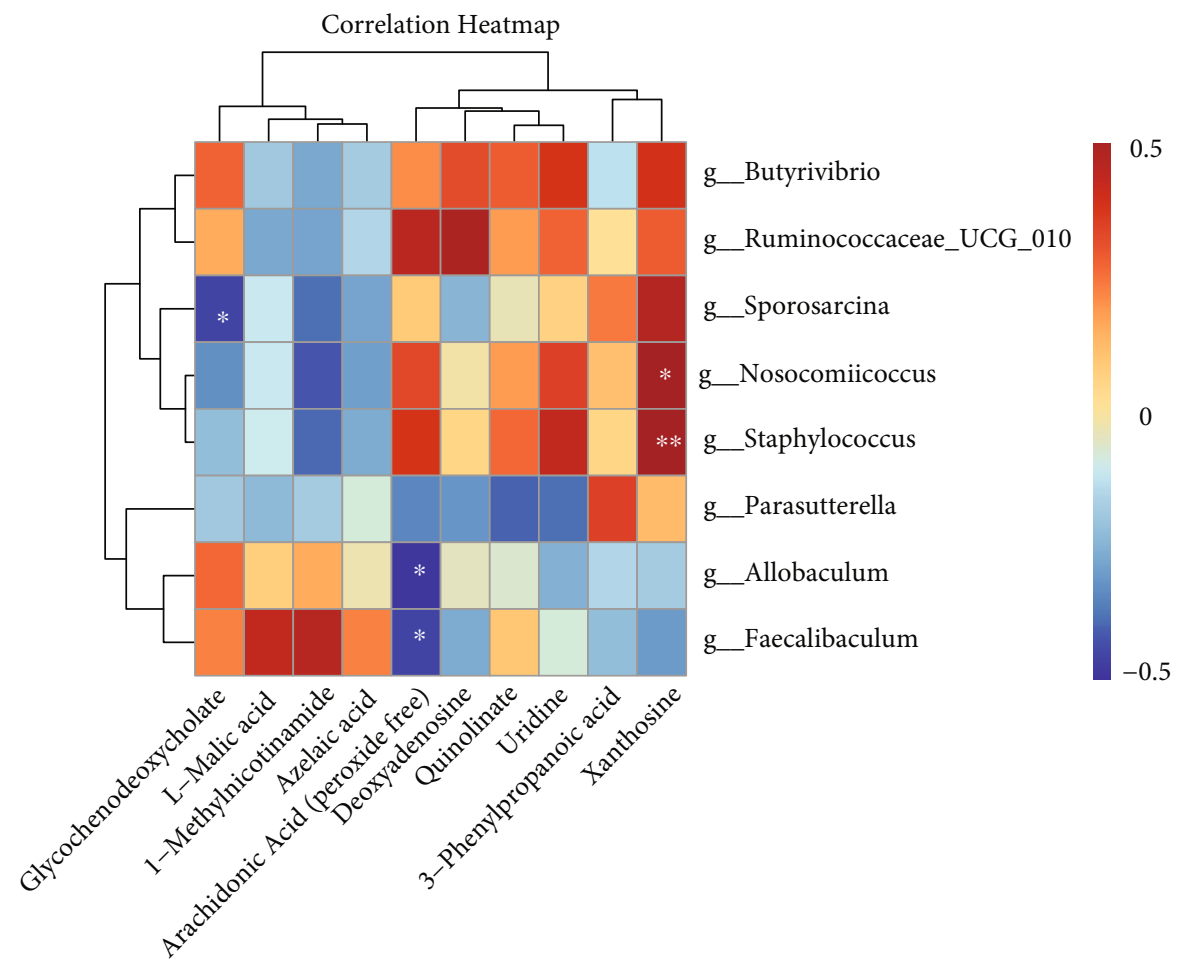

FIGURE 12: A correlation heat map is used to represent relevance analysis between gut microbiota and fecal biomarkers.

metabolism, purine metabolism, citrate cycle (TCA cycle), pyruvate metabolism, arachidonic acid metabolism, pyrimidine metabolism, and primary bile acid biosynthesis.

Arachidonic acid is an essential fatty acid in the body and is also a precursor to enzymatic and nonenzymatic oxidation products such as prostaglandins, thromboxanes, leukotrienes, lipoxygenins, and isoprostane, which can exert oxidative stress signals associated with inflammation [44]. Studies have shown that after the action of 5-lipoxygenase, arachidonic acid can be converted into leukotriene A4, which is catalyzed by hydrolase and has an inhibitory effect on the immune response and the formation of lymphokines [45]. In addition, studies have shown that epoxyeicosatrienoic acid produced from arachidonic acid can exert antiinflammatory effects by inhibiting the release of inflammatory factors such as IL-6, TNF- $\alpha$, and IL- $\beta$ [46]. In this study, arachidonic acid was significantly higher in the ZBVO administration group compared to the MC group, suggesting that ZBVO may exert a regulatory effect on STC by modulating arachidonic acid metabolism to enhance antiinflammatory effects in vivo.

Purine metabolism, TCA cycle, and pyruvate metabolism are all closely related to energy metabolism. Increased energy metabolism in the rat intestine plays an important role in regulating STC, as the peristalsis of the intestine requires a large amount of energy. Purines are among the most abundant metabolites in mammalian cells, and in addition to producing DNA and RNA molecules, purine nucleotides such as adenosine $5^{\prime}$ triphosphate (TP) and guanosine $5^{\prime}$ triphosphate (GTP) are critical for providing cellular energy and intracellular signals, respectively [47]. In this experiment, the loss of appetite and sluggish locomotion in STC rats may be related to the decrease of xanthine nucleosides and thus the decreased energy metabolism, meaning the purine-related xanthine nucleoside is in a disorder in STC rats. After ZBVO regulation, xanthine nucleoside was backregulated, which promoted the recovery of purine metabolism disorder. The citrate cycle is the final metabolic pathway for three major nutrients (amino acids, sugars, and lipids) and is the hub of energy metabolism. L-Malate is an intermediate in the tricarboxylic acid cycle and pyruvate metabolism. In this study, the level of L-malic acid in the MC group was reduced, suggesting that their energy metabolism was disturbed. While $\mathrm{ZBVO}$ regulated the TCA cycle, the pyruvate metabolism pathway increased L-malic acid in STC rats, thus providing sufficient energy for intestinal motility. Bile acid has a regulatory effect on gastrointestinal motility and promotes the digestion and absorption of lipids in food [48]. In the case of gastrointestinal motility disorders, such as STC, the hepatic and intestinal circulation of bile acids is affected. Arachidonic acid is a product of cholesterol in the presence of $7 \alpha$ hydroxylase, and here, the level of glycocholic acid in the feces in the MC group was significantly reduced, suggesting that primary bile acid biosynthesis was disrupted by the diphenoxylate tablets. In contrast, the administration of $\mathrm{ZBVO}$ resulted in a significant increase in arachidonic acid, suggesting that ZBVO regulates primary bile acid biosynthesis, which may explain the rheumatological, spleen-building, and digestive effects of ZBVO. 


\section{Conclusion}

This study demonstrated the alleviating effect of transdermal administration of ZBVO on constipation in STC rats. ZBVO can promote intestinal peristalsis, increase fecal water content, regulate gastrointestinal hormone level (GAS and SP), reduce the inflammatory response (IL-6 and TNF- $\alpha$ ), and regulate brain-intestinal peptides (5-HT and VIP) after transdermal administration. In addition, the composition of the intestinal microbiota is improved by ZBVO's reduction of harmful bacteria and promotion of beneficial bacteria. This study may provide efficacious drugs for STC patients. This study explores a new drug delivery component and drug delivery route for ZS-BZ-regulated STC and provides a new direction for the development of aromatic drug macrohealth products.

\section{Data Availability}

All data used to support the findings of this study are available from the corresponding author upon request.

\section{Ethical Approval}

The animal study was reviewed and approved by the Animal Ethics Committee of Jiangxi University of Traditional Chinese Medicine.

\section{Conflicts of Interest}

The authors declare that there is no conflict of interests regarding the publication of this paper.

\section{Authors' Contributions}

MY and FW cosupervised the study. LFW, XFZ, and FW codesigned the study. FW and QYC codesigned and implemented the therapeutic measures and sampling. LFW and HTL analyzed the data and made the plots and tables of the result. LFW, MY, FQL, and FW wrote and revised the manuscript, and all authors reviewed and improved the manuscript. Liangfeng Wang is the first author. Fang Wang is the co-first author.

\section{Acknowledgments}

This study was supported by grants from the Project of National Natural Science Foundation of China (81960714), Jiangxi Province Science and Technology Major Projects (20194ABC28009), and the funding from Jiangxi University of Traditional Chinese Medicine (JXSYLXK-ZHYAO083 and JXSYLXKZHYAO084).

\section{Supplementary Materials}

Supplementary Figure 1: plot of sample dilution curves and Shannon-Wiener curves for operable taxonomic units in the population. Supplemental Table 1: elution gradient. (Supplementary Materials)

\section{References}

[1] U. Hayat, M. Dugum, and S. Garg, "Chronic constipationupdate on management," Cleveland Clinic Journal of Medicine, vol. 84, no. 5, pp. 397-408, 2017.

[2] H. L. Wang, "Understanding the pathogenesis of slow-transit constipation: one step forward," Digestive Diseases and Sciences, vol. 60, no. 8, pp. 2216-2218, 2015.

[3] M. Camilleri, A. C. Ford, G. M. Mawe et al., "Chronic constipation," Nature Reviews Disease Primers, vol. 3, no. 1, 2017.

[4] H. Chu, L. Zhong, H. Li, X. Zhang, J. Zhang, and X. Hou, "Epidemiology characteristics of constipation for general population, pediatric population, and elderly population in China," Gastroenterology Research and Practice, vol. 2014, Article ID 532734, 11 pages, 2014.

[5] T. Stern and A. M. Davis, "Evaluation and treatment of patients with constipation," JAMA, vol. 315 , no. 2, pp. 192193, 2016.

[6] V. C. Costilla and A. E. Foxx-Orenstein, "Constipation: Understanding Mechanisms and Management," Clinics in Geriatric Medicine, vol. 30, no. 1, pp. 107-115, 2014.

[7] G. Bassotti and C. Blandizzi, "Understanding and treating refractory constipation," World Journal of Gastrointestinal Pharmacology and Therapeutics, vol. 5, no. 2, pp. 77-85, 2014.

[8] Y. Zeng, X. Zhang, J. Zhou, X. Wang, R. Jiao, and Z. Liu, “Efficacy of electroacupuncture compared with transcutaneous electric nerve stimulation for functional constipation," Medicine, vol. 97, no. 19, article e692, 2018.

[9] K. Takayama, C. Takahara, N. Tabuchi, and N. Okamura, "Daiokanzoto (Da-Huang-Gan-Cao-Tang) is an effective laxative in gut microbiota associated with constipation," Scientific Reports, vol. 9, no. 1, p. 3833, 2019.

[10] M. J. He, J. X. Yang, Y. Wei, and J. An, "Effects of Fructus Aurantii Immaturus volatile oil on slow transit constipation in rats," China Journal of Traditional Chinese Medicine and Pharmacy, vol. 28, no. 5, pp. 1487-1491, 2013.

[11] M. D. S. Costa, J. E. Rocha, F. Fábia et al., "Comparative analysis of the antibacterial and drug-modulatory effect of d-limonene alone and complexed with $\beta$-cyclodextrin," European Journal of Pharmaceutical Sciences, vol. 11, no. 36, pp. 1-16, 2018.

[12] H. P. Chen and W. Z. Wu, "Research progress of Atractylodes macrocephala," Guangdong Pharmacy, no. 5, pp. 19-21, 2002.

[13] H. Wang, N. Yang, H. Q. Lin, Q. H. Dong, and P. Y. Li, "Research progress on Atractylodes macrocephala koidz of the chemical composition, pharmacological effects and their clinical application," Gansu Medical Journal, vol. 37, no. 1, pp. 23-26, 2018.

[14] W. B. Han, "Clinical observation of acupoint application for slow transit constipation due to spleen deficiency and intestine dryness," Shanghai Journal of Acupuncture and Moxibustion, vol. 37, no. 12, pp. 1359-1362, 2018.

[15] C. Zhang, W. Gong, Z. Li, D. Gao, and Y. Gao, "Research progress of gut flora in improving human wellness," Food Science and Human Wellness, vol. 8, no. 2, pp. 102-105, 2019.

[16] S. B. Bhardwaj, "Gut flora and its modification as a therapy," Reviews in Medical Microbiology, vol. 24, no. 3, pp. 52-54, 2013.

[17] B. Y. Bai, X. Y. Xin, Q. Zhou et al., "Dietary fiber of red jujube ameliorates functional constipation and improves intestinal health in mice," Food Science, vol. 37, no. 23, pp. 254-259, 2016. 
[18] S. Estruel-Amades, M. Massot-Cladera, F. Pérez-Cano, À. Franch, M. Castell, and M. Camps-Bossacoma, "Hesperidin effects on gut microbiota and gut-associated lymphoid tissue in healthy rats," Nutrients, vol. 11, no. 2, p. 324, 2019.

[19] Z. Wen, M. He, C. Peng et al., "Metabolomics and 16S rRNA gene sequencing analyses of changes in the intestinal flora and biomarkers induced by gastrodia-uncaria treatment in a rat model of chronic migraine," Frontiers in Pharmacology, vol. 10, 2019.

[20] G. J. Peng, B. Y. Shi, J. S. Tian, S. Gao, and X. M. Qin, " ${ }^{1} H$ NMR based metabonomics study on the antidepressant effect of genipin in rat hippocampus," Acta Pharmaceutica Sinica, vol. 49, no. 2, pp. 209-216, 2014.

[21] Y. Zhan, X. Tang, H. Xu, and S. Tang, "Maren pills improve constipation via regulating AQP3 and NF- $\kappa$ B signaling pathway in slow transit constipation in vitro and in vivo," Evidence-based Complementary and Alternative Medicine, vol. 2020, Article ID 9837384, 12 pages, 2020.

[22] X. Ren, L. Liu, Y. Gamallat, B. Zhang, and Y. Xin, "Enteromorpha and polysaccharides from enteromorpha ameliorate loperamide- induced constipation in mice," Biomedicine \& Pharmacotherapy, vol. 96, pp. 1075-1081, 2017.

[23] H. Tian, X. Ge, Y. Nie et al., "Fecal microbiota transplantation in patients with slow-transit constipation: a randomized, clinical trial," PLoS One, vol. 12, no. 2, article e171308, 2017.

[24] H. Xu, Y. Zhang, Z. Liu et al., "ETCM: an encyclopaedia of traditional Chinese medicine," Nucleic Acids Research, vol. 47, no. D1, pp. D976-D982, 2019.

[25] H. Xu, Y. Zhang, P. Wang et al., "A comprehensive review of integrative pharmacology-based investigation: a paradigm shift in traditional Chinese medicine," Acta Pharmaceutica Sinica B, vol. 11, no. 6, pp. 1379-1399, 2021.

[26] W. X. Xia, X. S. Zhang, and T. Liang, "Modern research on Aurantii Fructus Immaturus and Atractylodis Macrocephalae Rhizoma and their compatibility," Information on Traditional Chinese Medicine, vol. 29, no. 3, pp. 15-19, 2012.

[27] F. Bonamin, T. M. Moraes, R. C. dos Santos et al., "The effect of a minor constituent of essential oil from Citrus aurantium: The role of $\beta$-myrcene in preventing peptic ulcer disease," Chemico-Biological Interactions, vol. 212, pp. 11-19, 2014.

[28] A. L. Rozza and C. H. Pellizzon, "Essential oils from medicinal and aromatic plants: a review of the gastroprotective and ulcer-healing activities," Fundamental \& Clinical Pharmacology, vol. 27, no. 1, pp. 51-63, 2013.

[29] Y. Zheng, S. Wang, X. S. Meng, and Y. R. Bao, "Analysis of the orange essential oil by GC-MS and research on the prokinetic effect of it," Lishizhen Medicine and Materia Medica Research, vol. 26, no. 3, pp. 516-518, 2015.

[30] L. F. Wang, X. L. Liu, H. T. Li et al., "Mechanism of Aurantii Fructus Immaturus volatile oil in treatment of slow transit constipation based on network pharmacology," China Journal of Chinese Materia Medica, vol. 45, no. 8, pp. 1909-1917, 2020.

[31] H. Jiang, J. Dong, S. Jiang et al., "Effect of Durio zibethinus rind polysaccharide on functional constipation and intestinal microbiota in rats," Food Research International, vol. 136, article 109316, 2020.

[32] M. R. Hellmich, X. L. Rui, H. L. Hellmich, R. Y. D. Fleming, B. M. Evers, and C. M. Townsend Jr., "Human Colorectal Cancers Express a Constitutively Active Cholecystokinin-B/Gastrin Receptor That Stimulates Cell Growth," Journal of Biological Chemistry, vol. 275, no. 41, pp. 32122-32128, 2000.
[33] D. Y. Hwang, S. Kim, and H. S. Hong, "Substance-p ameliorates dextran sodium sulfate-induced intestinal damage by preserving tissue barrier function," Tissue Engineering and Regenerative Medicine, vol. 15, no. 1, pp. 63-73, 2018.

[34] F. Liu, X. P. Wei, and X. G. Tang, "Clinical efficacy of modified Zhishi Daozhiwan on slow transit constipation," Chinese Journal of Experimental Traditional Medical Formulae, vol. 26, no. 2, pp. 92-97, 2020.

[35] E. A. Mayer, "Gut feelings: the emerging biology of gut-brain communication," Nature Reviews Neuroscience, vol. 12, no. 8, pp. 453-466, 2011.

[36] X. Zhou, H. H. Qian, D. Zhang, and L. Zeng, "Study on brainintestinal theory of traditional Chinese medicine based on mechanism of interaction between gut microbiota and braingut axis," Chinese Archives of Traditional Chinese Medicine, vol. 38, no. 3, pp. 119-122, 2020.

[37] M. D. Gershon, "5-Hydroxytryptamine (serotonin) in the gastrointestinal tract," Current Opinion in Endocrinology \& Diabetes and Obesity, vol. 20, no. 1, pp. 14-21, 2013.

[38] G. Bassotti, G. de Roberto, D. Castellani, L. Sediari, and A. Morelli, "Normal aspects of colorectal motility and abnormalities in slow transit constipation," World Journal of Gastroenterology, vol. 11, no. 18, pp. 2691-2696, 2005.

[39] J. M. Yano, K. Yu, G. P. Donaldson et al., "Indigenous bacteria from the gut microbiota regulate host serotonin biosynthesis," Cell, vol. 161, no. 2, pp. 264-276, 2015.

[40] J. Nzakizwanayo, C. Dedi, G. Standen, W. M. Macfarlane, B. A. Patel, and B. V. Jones, "Escherichia coli Nissle 1917 enhances bioavailability of serotonin in gut tissues through modulation of synthesis and clearance," Scientific Reports, vol. 5, no. 1, 2015.

[41] Y. C. Dou, The effects of brain gut peptide and anxiety /depression on anorectal dynamics in elderly patients with constipation, vol. Master, p. 47, 2018.

[42] A. B. Shreiner, J. Y. Kao, and V. B. Young, "The gut microbiome in health and in disease," Current Opinion in Gastroenterology, vol. 31, no. 1, pp. 69-75, 2015.

[43] A. Vich Vila, F. Imhann, V. Collij et al., "Gut microbiota composition and functional changes in inflammatory bowel disease and irritable bowel syndrome," Science Translational Medicine, vol. 10, no. 472, 2018.

[44] A. Trostchansky, I. Wood, and H. Rubbo, "Regulation of arachidonic acid oxidation and metabolism by lipid electrophiles," Prostaglandins \& Other Lipid Mediators, vol. 152, article 106482, 2021.

[45] B. Yan, H. Chu, D. Yang et al., "Characterization of the lipidomic profile of human coronavirus-infected cells: implications for lipid metabolism remodeling upon coronavirus replication," Viruses, vol. 11, no. 1, p. 73, 2019.

[46] X. C. Yan, W. N. Mu, Y. Qiang et al., "CYP450 epoxygenase/ EET pathway attenuates adipose inflammation of obese mice through HIF-1 $\alpha$," Chinese Journal of Pathophysiology, vol. 36, no. 9, pp. 1661-1666, 2020.

[47] A. M. Pedley and S. J. Benkovic, "A new view into the regulation of purine metabolism: the purinosome," Trends in Biochemical Sciences, vol. 42, no. 2, pp. 141-154, 2017.

[48] R. Ma, Q. Xie, J. Wang et al., "Effect of compatibility of alcohol extract of Magnoliae Officinalis Cortex and alcohol extract of Polygalae Radix on fecal metabolites of rats," Chinese Journal of Experimental Traditional Medical Formulae, vol. 25, no. 11 , pp. 1-7, 2019. 